MODELING, IDENTIFICATION AND CONTROL, 1988, VOL. 9, NO. 1, 17-45

doi:10.417//mic.1988.12

\title{
Identification of layers in oil-tanks using a reflectometer
}

\author{
ANTON M. BØIFOT $\dagger$
}

Keywords: Frequency domain analyses, time domain analyses, windows, dielectrical properties, attenuation, methods of detection, simulations, practical measurements.

This paper treats the possibilities of analysing layers in oil-tanks using a reflectometer. First some basic principles and expressions are presented. After that examples of commonly used windows are shown and a new generation of windows developed. These are called Hammingfolded windows, and can have side lobe levels below $-85 \mathrm{~dB}$.

The simulations treat different kinds of detections, particularly the possibilities of separating echoes of unequal strength. Important results are the use of windows and sometimes post-signal processing. These methods can either be used separately or in a combined version. The simulations also include studies of the electrical properties of unrefined oil, water and waxed oil (mud).

The practical measurements demonstrate the use of windows and further signal processing. This is done by simulating the conditions in an oil-tank by means of an extended coaxial line. In this line different kinds of reflectors as well as unrefined oil and water are used to obtain practical echoes.

\section{Introduction}

For measurements in media with losses and dispersion it can be very convenient to use electromagnetic waves. Examples of appropriate applications are the characterization of geological structures and liquids with different layers. This paper focuses particularly on the possibilities for the evaluation of layers in oil-tanks using the reflectometer principle.

The different layers are characterized by the registration of discontinuations in the dielectric constant. One way of doing this is to use a system based on real time domain pulses, and a lot of work has been done to develop equipment for geological purposes based on this principle. Another way is to utilize synthetical pulses and the reflectometer principle. No description of such equipment for analysing different layers in oil-tanks has been found in the literature.

\section{Basic principle}

The basic principle of this radar system is to transmit a pulse from level $Z_{0}$ (Fig. 1), and then measure the elapsed time between the transmitted and the different reflected signals.

The pulse is constructed so as to be synthetical in the frequency domain (Fig. 2). First a signal with the frequency $f_{1}$ is transmitted, and the equipment registers the amplitude and phase of the reflection coefficient. Then a signal with frequency $f_{2}$ is transmitted, and the same procedure is repeated.

Received August 261987.

† ELAB, 7034 Trondheim, Norway. 


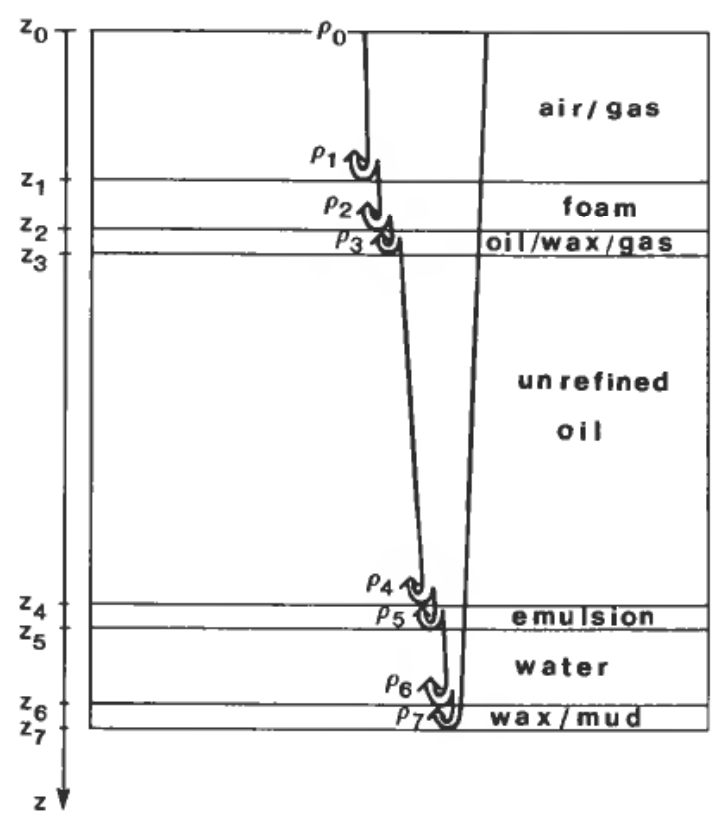

Figure 1. Model of the tank.

The synthetical pulse is complete when the amplitude and phase of all the $N$ frequencies are registered. To get the time domain the synthetical pulse had to be subjected to a Fourier-transformation.

With a sensible choice of centre frequency, bandwidth and number of frequencies, it is possible to measure the time of the arrival of the signals $\rho_{1}, \rho_{2}, \ldots$, $\rho_{7}$. In order to calculate the range of the different impedance transitions it is necessary to know the dielectric constant of the media.

\section{Basic expressions}

The notation for some of the parameters used in this paper is:

$\alpha \quad$ attenuation constant

$\beta \quad$ phase constant

$\rho \quad$ reflection coefficient

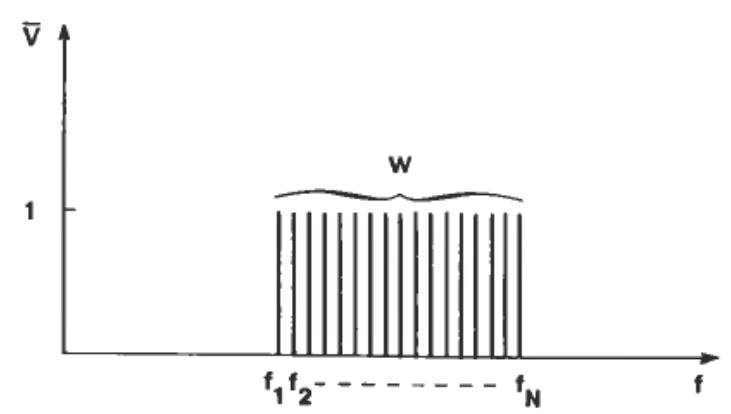

Figure 2. Model of the pulse. 


$\begin{array}{ll}f & \text { frequency } \\ t & \text { time } \\ z & \text { range } \\ \varepsilon=\varepsilon^{\prime}-j \varepsilon^{\prime \prime} & \text { dielectric constant } \\ \varepsilon_{0} & \text { dielectric constant in vacuum } \\ f_{c} & \text { centre frequency } \\ w & \text { bandwidth } \\ \tau & \text { time for arrival } \\ c & \text { velocity of the light } \\ k \Delta f & \text { discrete frequency variable } \\ i \Delta t & \text { discrete time variable } \\ m \Delta t & \text { discrete time for arrival }\end{array}$

\subsection{One impedance transition}

The model for one impedance transition is shown in Fig. 3. The reflection coefficient at range $Z_{1}$ is assumed to be independent of the frequency:

$$
\rho_{1}=r_{1} \exp \left(j \phi_{1}\right)
$$

In the analogue case this leads to the following expression for the reflection coefficient in the frequency domain:

$$
\begin{aligned}
\rho_{0}(f, \tau) & =r_{1} \exp \left(-2 \alpha_{0} Z_{1}+j \phi_{1}\right) \exp (-j 2 \pi f \tau) \operatorname{rect}\left(\frac{f-f_{\mathrm{c}}}{w}\right) \\
\operatorname{rect}(f) & =\left\{\begin{array}{l}
1,|f| \leqslant \frac{1}{2} \\
0,|f|>\frac{1}{2}
\end{array}\right.
\end{aligned}
$$

The Fourier-transformation gives for the time domain:

$$
\begin{aligned}
& \rho_{0}(t, \tau)=r_{1} \exp \left(-2 \alpha_{0} Z_{1}\right) \exp \left(j\left(\phi_{1}+2 \pi f_{\mathrm{c}}(t-\tau)\right)\right) \operatorname{sinc}(w(t-\tau)) \\
& \operatorname{sinc} w t=\frac{\sin \pi w t}{\pi w t}
\end{aligned}
$$

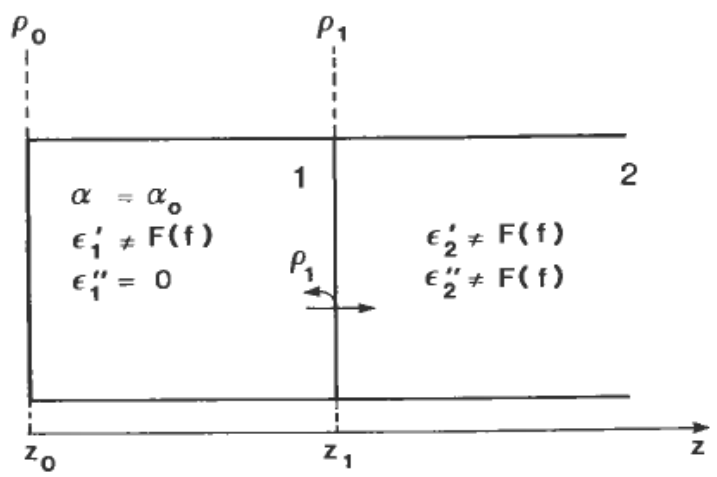

Figure 3. Dielectric model. 


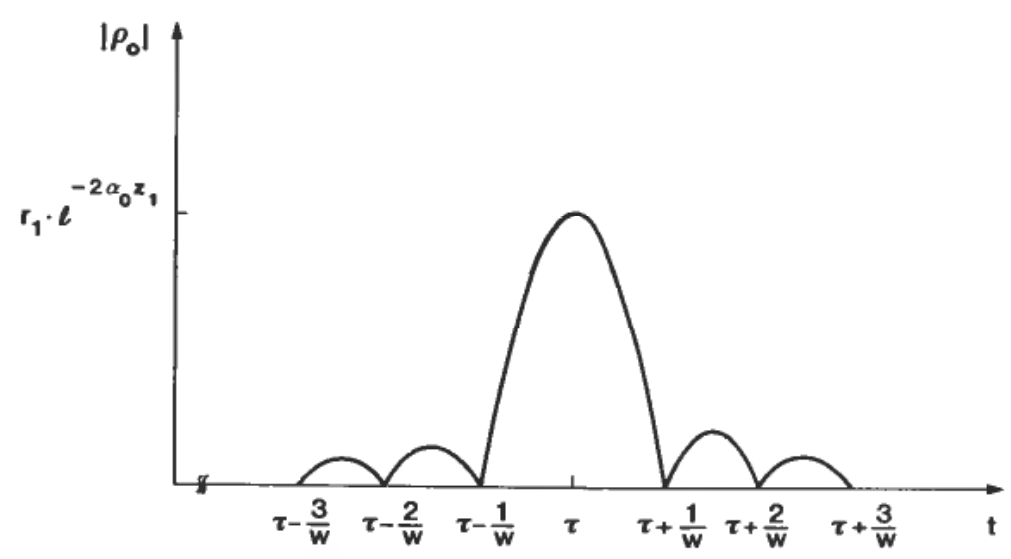

Figure 4. Reflection coefficient in the time domain.

This is a sinc-function centred around the time $\tau$. The addition to the phase at the range $Z_{1}$ does not affect the time for the arrival of the pulse $\left(\phi_{1}\right.$ independent of the frequency), see Fig. 4.

The connection between the time domain and the range is very simple:

$$
Z_{1}=\frac{\tau \times c}{2 \sqrt{\left(\varepsilon^{\prime} / \varepsilon_{0}\right)}}
$$

The distance from $Z_{1}$ to the first zero:

$$
\Delta Z=\frac{c}{2 w \sqrt{\left(\varepsilon^{\prime} / \varepsilon_{0}\right)}}
$$

In the discrete case, the analogous expressions are:

$$
\begin{aligned}
& \rho_{0}(k, m)=r_{1} \exp \left(-2 \alpha_{0} Z_{1}+j \phi_{1}\right) \exp (-j 2 \pi(k m / N)) \operatorname{rect}\left(\frac{k-N_{0}}{N-1}\right) \\
& f_{c}=\Delta f \times N_{0}, \quad N_{0}: \text { integer } \\
& \rho_{0}(i, m)=\frac{r_{1}}{N} \exp \left(-2 \alpha_{0} Z_{1}+j \phi_{1}\right) \exp \left(j(2 \pi / N)(i-m) N_{0}\right) \frac{\sin \pi(i-m)}{\sin [(\pi / N)(i-m)]}
\end{aligned}
$$

Transformation to the time domain:

$$
\begin{aligned}
t & =i / w \\
\tau & =m / w
\end{aligned}
$$

Equation (6) is periodic with the period:

$$
Z_{\max }=\frac{c}{2 \sqrt{\left(\varepsilon^{\prime} / \varepsilon_{0}\right)}} \frac{N}{w}
$$

The envelope of Eqn (6) in the time domain:

$$
\left|\rho_{0}(t, \tau)\right|=\frac{r_{1}}{N} \exp \left(-2 \alpha_{0} Z_{1}\right) \frac{\sin \pi w(t-\tau)}{\sin (\pi / N) w(t-\tau)}
$$

The main difference between the analogue and the discrete case is that the discrete reflection coefficient is periodic and has a slightly different envelope. 


\subsection{Several impedance transitions}

In a practical oil-tank there will probably be three or more impedance transitions. This will make the situation much more difficult to analyse mathematically, see Fig. 5.

If $Q$ impedance transitions and only the echoes between transition $q$ and $q-1$ are taken into account, then the simplified expression for $|\rho(z)|$ is:

$$
|\rho(z)|=\left|\rho_{1}^{0}\right| \operatorname{sinc}\left(\frac{Z-Z_{1}}{\Delta Z}\right)+\sum_{p=0}^{\infty} \sum_{q=2}^{Q}\left|\rho_{q}^{p}\right| \operatorname{sinc} \frac{Z-\left(Z_{q}+p\left(Z_{q}-Z_{q-1}\right)\right)}{\Delta Z}
$$

As Eqn (8) shows the expression which describes the tank are quickly becoming complicated, and the conditions in the tank have been simulated on a computer to simplify the analysis.

\section{Windows}

In Fig. 5 it is supposed that all of the main echoes are stronger than the level of the side lobes. Since in a practical tank this is not the case, it is more convenient to use window functions in order to lower the side lobes.

\subsection{Common windows}

Some common windows which are used in this work are listed below:

\section{Rectangular:}

$$
w(k)=1, \quad 0 \leqslant k \leqslant N-1
$$

Barlett

$$
w(k)=\left\{\begin{array}{cl}
\frac{2 k}{n-1}, & 0 \leqslant k \leqslant \frac{N-1}{2} \\
2-\frac{2 k}{N-1}, & \frac{N-1}{2} \leqslant k \leqslant N-1
\end{array}\right.
$$

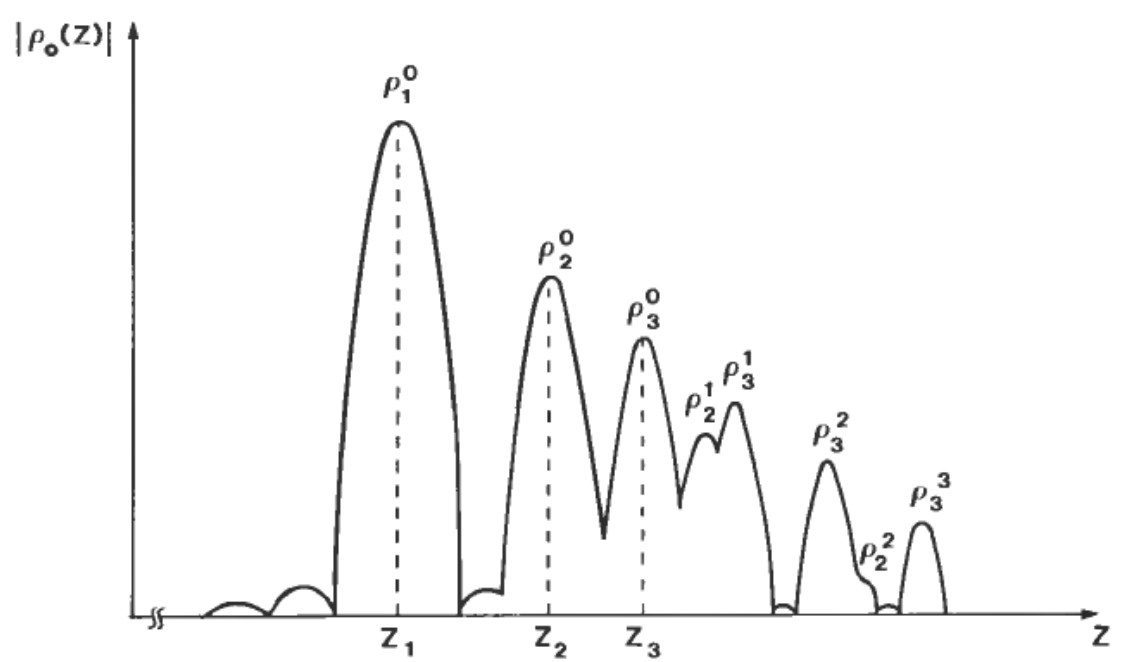

Figure 5. Multiple reflections, $Q=3$. 
Hanning

$$
w(k)=0.5-0.5 \cos \left(\frac{2 \pi k}{N-1}\right), \quad 0 \leqslant k \leqslant N-1
$$

Hamming

$$
w(k)=0.54-0.46 \cos \left(\frac{2 \pi k}{N-1}\right), \quad 0 \leqslant k \leqslant N-1
$$

Blackman

$$
w(k)=0.42-0.5 \cos \left(\frac{2 \pi k}{N-1}\right)+0.08 \cos \left(\frac{4 \pi k}{N-1}\right), \quad 0 \leqslant k \leqslant N-1
$$

The Fourier-transformation gives:

Rectangular :

$$
w(i)=\frac{\sin \pi i}{N \sin (\pi / N) i}
$$

Barlett

$$
w(i)=2 \times\left[\frac{\sin (\pi / 2) i}{N \sin (\pi / N) i}\right]^{2}
$$

Hanning

$$
w(i)=\frac{0.5 \sin \pi i}{N \sin (\pi / N) i}+\frac{0.25 \sin \pi(i-1)}{N \sin (\pi / N)(i-1)}+\frac{0.25 \sin \pi(i+1)}{N \sin (\pi / N)(i+1)}
$$

Hamming

$$
w(i)=\frac{0.54 \sin \pi i}{N \sin (\pi / N) i}+\frac{0.23 \sin \pi(i-1)}{N \sin (\pi / N)(i-1)}+\frac{0.23 \sin \pi(i+1)}{N \sin (\pi / N)(i+1)}
$$

Blackman

$$
\begin{aligned}
w(i)= & \frac{0.42 \sin \pi i}{N \sin (\pi / N) i}+\frac{0.25 \sin \pi(i-1)}{N \sin (\pi / N)(i-1)}+\frac{0.25 \sin \pi(i+1)}{N \sin (\pi / N)(i+1)} \\
& -\frac{0.04 \sin \pi(i-2)}{N \sin (\pi / N)(i-2)}-\frac{0.04 \sin \pi(i+2)}{N \sin (\pi / N)(i+2)}
\end{aligned}
$$

These transformations are shown in Figures 6 and 7.

\subsection{Generalized Hammingfolded window}

A practical reflectometer system may be capable of distinguishing echoes with a difference of approximately $80 \mathrm{~dB}$. To take advantage of the whole capacity it is necessary to have windows with side lobes which have a level of less than $-80 \mathrm{~dB}$. For this purpose a new generation of windows called generalized Hammingfolded 


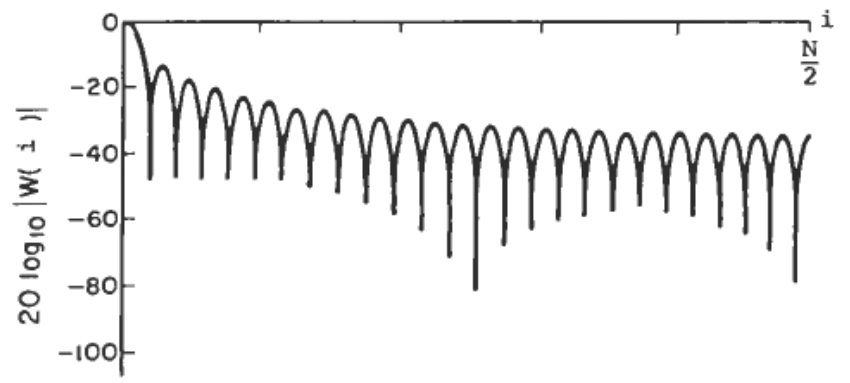

(a)

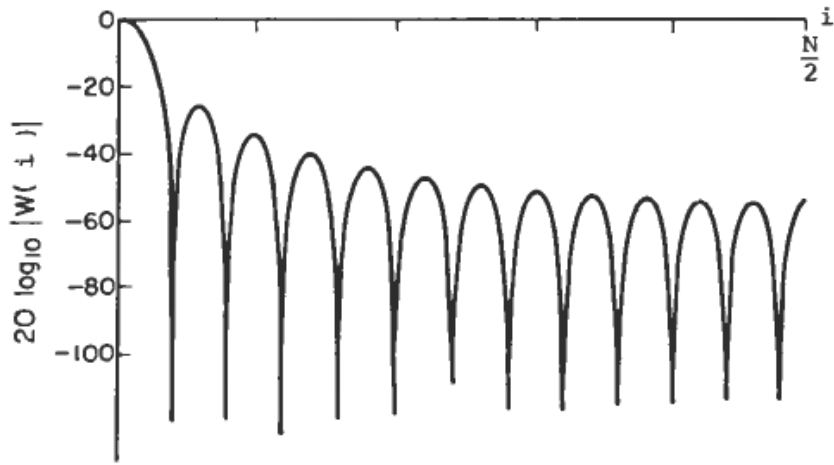

(b)

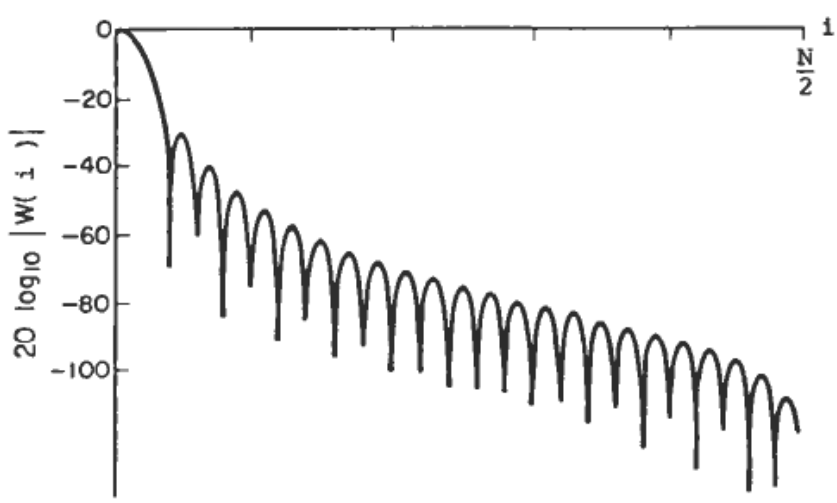

(c)

Figure 6. Discrete Fourier Transforms (DFT) of windows. (a) Rectangular, (b) Barlett, (c) Hanning.

windows has been developed. They are constructed by folding a Hamming window by itself:

$$
\begin{aligned}
& 0 \leqslant f \leqslant \frac{w}{2}: \quad w(f)=\int_{0}^{f}\left(h-(1-h) \cos \frac{4 \pi \tau}{w}\right)\left(h-(1-h) \cos \frac{4 \pi}{w}(f-\tau)\right) d \tau \\
& \frac{w}{2}<f \leqslant w: \quad w(f)=\int_{f-(w / 2)}^{w / 2}\left(h-(1-h) \cos \frac{4 \pi \tau}{w}\right)\left(h-(1-h) \cos \frac{4 \pi}{w}(f-\tau)\right) d \tau \\
& h: \text { constant }
\end{aligned}
$$




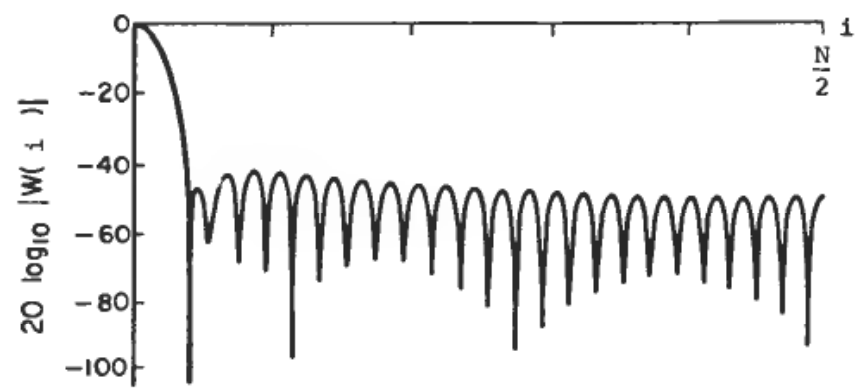

(a)

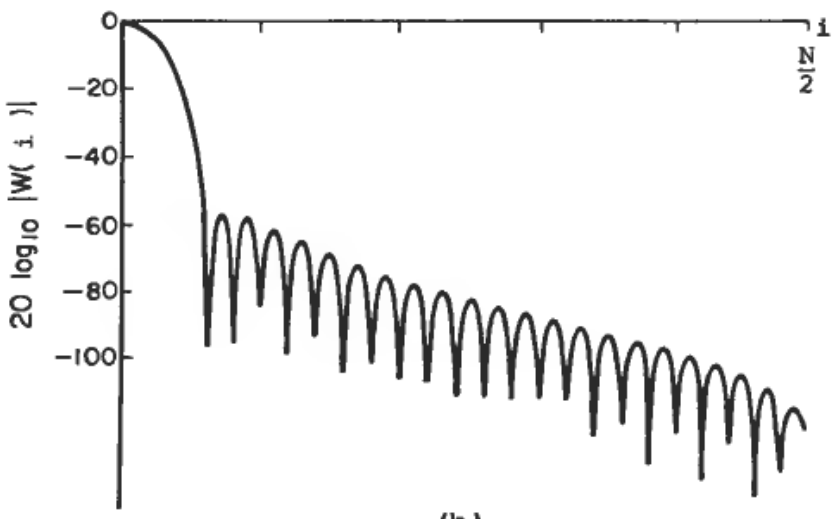

(b)

Figure 7. Discrete Fourier Transforms (DFT) of windows. (a) Hamming, (b) Blackman.

In the analogue case the generalized Hammingfolded window becomes:

Frequency domain:

$0 \leqslant f \leqslant \frac{w}{2}: \quad w(f)=\frac{4 h^{2} f+\frac{w(1-h)(1-5 h)}{2 \pi} \sin \frac{4 \pi f}{w}+2 f(1-h)^{2} \cos \frac{4 \pi f}{w}}{w\left(3 h^{2}-2 h+1\right)}$

$\frac{w}{2}<f \leqslant w: \quad w(f)=\left(4 h^{2} w+2 w(1-h)^{2} \cos \frac{4 \pi f}{w}-4 h^{2} f-\frac{w(1-h)(1-5 h)}{2 \pi} \sin \frac{4 \pi f}{w}\right.$

$$
\left.-2 f(1-h)^{2} \cos \frac{4 \pi f}{w}\right) \frac{1}{w\left(3 h^{2}-2 h+1\right)}
$$

Time domain:

$$
\begin{aligned}
w(t) & =\frac{1}{3 h^{2}-2 h+1}\left[h \operatorname{sinc} \frac{w}{2} t+\frac{1-h}{2} \operatorname{sinc} \frac{1}{2}(w t-2)+\frac{1-h}{2} \operatorname{sinc} \frac{1}{2}(w t+2)\right]^{2} \\
h & =0.54 \text { gives Hammingfolded window } \\
h & =0.5 \text { gives Hanningfolded window }
\end{aligned}
$$


In the discrete case the expressions for the Hammingfolded window are:

Frequency domain:

$$
\begin{aligned}
& 0 \leqslant k \leqslant \frac{N-1}{2}: \\
& w(k)= \frac{1}{0.7948}\left[1.1664 \frac{k}{(N-1)}-\frac{0.391}{\pi} \sin \frac{4 \pi k}{(N-1)}+0.4232 \frac{k}{(N-1)} \cos \frac{4 \pi k}{(N-1)}\right] \\
& \frac{N-1}{2}<k \leqslant N-1: \\
& w(k)=\frac{1}{0.7948}\left[1.1664+0.4232 \cos \frac{4 \pi k}{(N-1)}-1.1664 \frac{k}{(N-1)}\right. \\
&\left.\quad+\frac{0.391}{\pi} \sin \frac{4 \pi k}{(N-1)}-0.4232 \frac{k}{(N-1)} \cos \frac{4 \pi k}{(N-1)}\right]
\end{aligned}
$$

Time domain:

$$
w(i)=\frac{4}{0.7948}\left[\frac{0.54 \sin (\pi / 2) i}{N \sin (\pi / N) i}+\frac{0.23 \sin (\pi / 2)(i-2)}{N \sin (\pi / N)(i-2)}+\frac{0.23 \sin (\pi / 2)(i+2)}{N \sin (\pi / N)(i+2)}\right]^{2}
$$

The Hamming and the Hanningfolded windows are shown in Fig. 8.

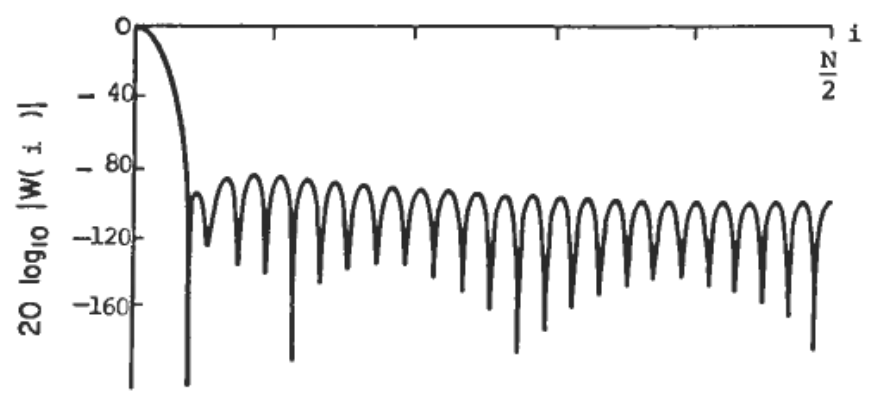

(a)

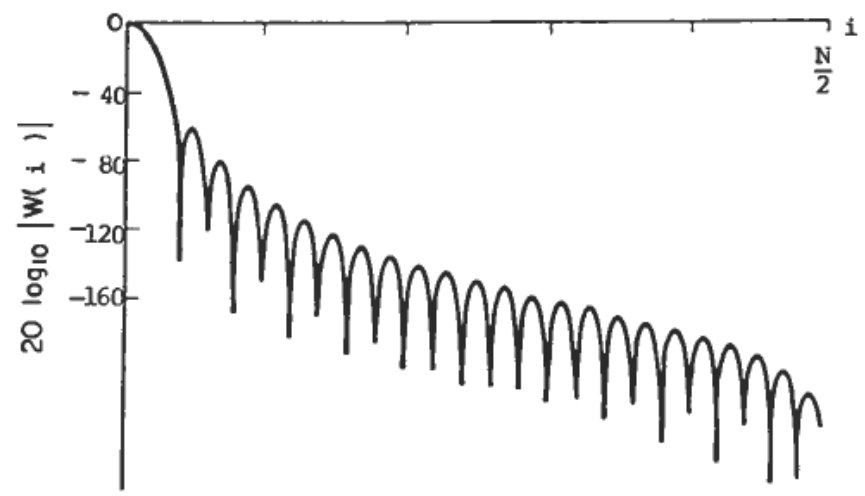

(b)

Figure 8. Discrete Fourier Transforms (DFT) of windows. (a) Hammingfolded window, (b) Hanningfolded window. 


\section{Electrical data for actual media}

In order to be able to simulate the conditions in the tank it is necessary to know the electrical data for the actual media. In this section, data for unrefined oil, water and waxed oil (mud) are presented. These are based on measurements carried out with a new method for measuring the dielectric constant of liquids using an automatic network analyser (described in a forthcoming paper by the present author). The calculations of the dielectric constant of water are also based on measurements described by Saxton and Lane (1952).

\subsection{Unrefined oil}

Figure 9 shows the relative real part of the dielectric constant. It drops weakly from approximately $2 \cdot 170$ to 2.145 in the frequency range from $300 \mathrm{MHz}$ to $12 \mathrm{GHz}$. Such behaviour is typical for liquids (von Hippel, 1952). In the same range the relative imaginary part falls from approximately $40 \times 10^{-3}$ to $9 \times 10^{-3}$, see Fig. 10. The value for the shaken sample is the highest. The reason for this is that different kinds of pollution are spread out in the oil by the shaking.

Figure 11 shows the attenuation as a function of the frequency. In a practical radar system there will be losses caused by reflections from the antenna, perhaps from foam, from the surface and attenuation owing to the antenna lobe. If we are not concerned with 'looking through' water and mud (wax) we can permit losses of $50 \mathrm{~dB}$, for instance. In a tank of 20-25 m we then can use frequencies up to about $2 \mathrm{GHz}$. A reasonable frequency bandwidth will then give total losses of approximately $50 \mathrm{~dB}$. If in this example we wanted to use $30 \mathrm{~dB}$ for the detection of water and mud, the tank cannot be deeper than about $10 \mathrm{~m}$. In the simulations the frequency bandwidth is very often $1.1-1.9 \mathrm{GHz}$. This covers many eventualities.

\subsection{Water}

5.2.1. Pure water In $\S 5.2$ and 5.3 the temperature $T$ is in ${ }^{\circ} \mathrm{C}$, the salt content $(\mathrm{NaCl}) S$ in $\mathrm{g} / \mathrm{l}$ and the frequency $F$ in $\mathrm{GHz}$. In $\S \S 5,6$ and 7 the curves are always

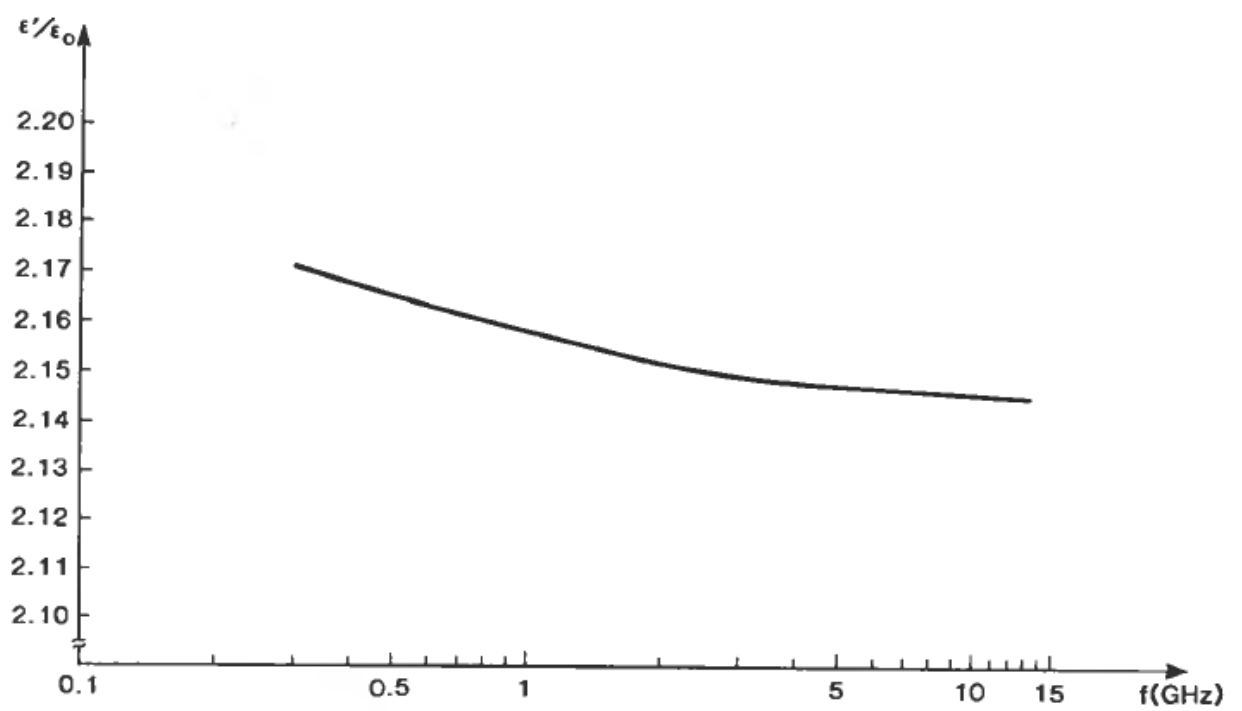

Figure 9. Unrefined oil, mean value of $\varepsilon^{\prime} / \varepsilon_{0}$. 


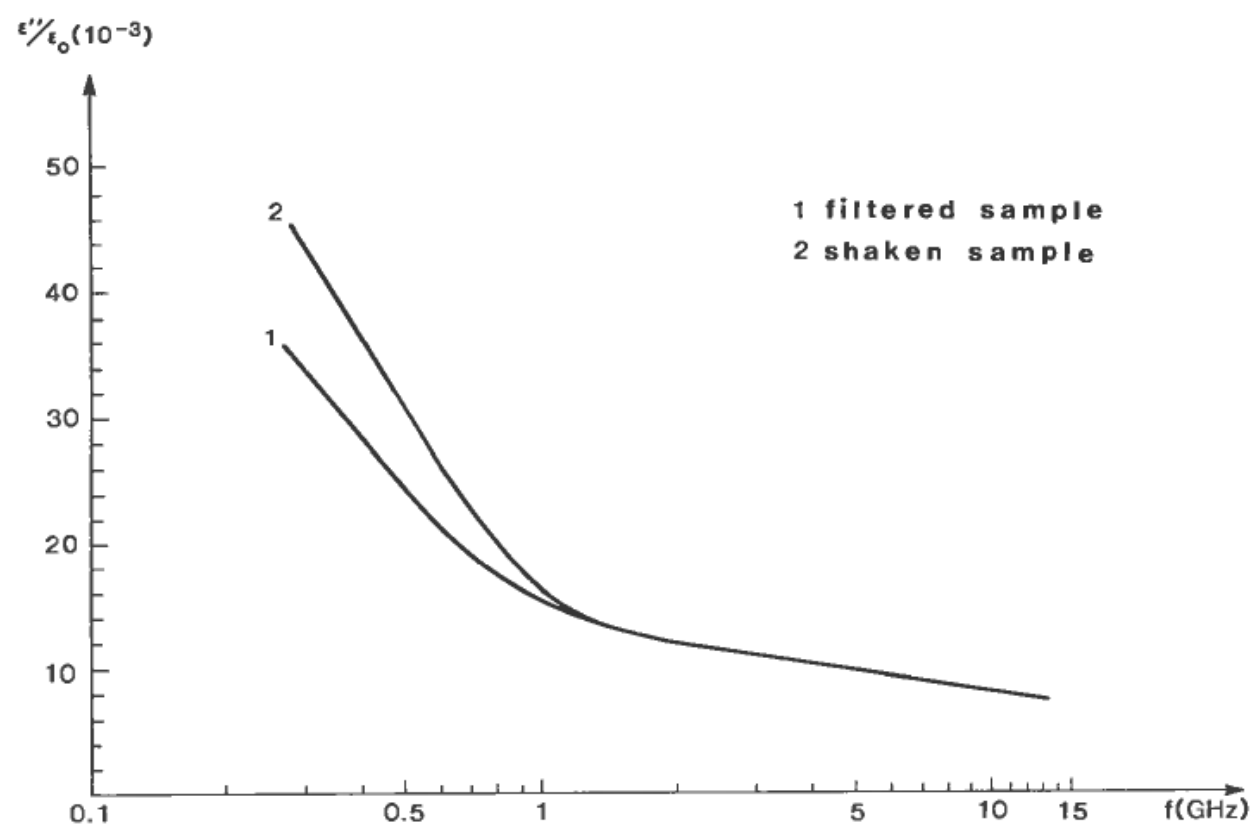

Figure 10. Unrefined oil, mean value of $\varepsilon^{\prime \prime} / \varepsilon_{0}$.

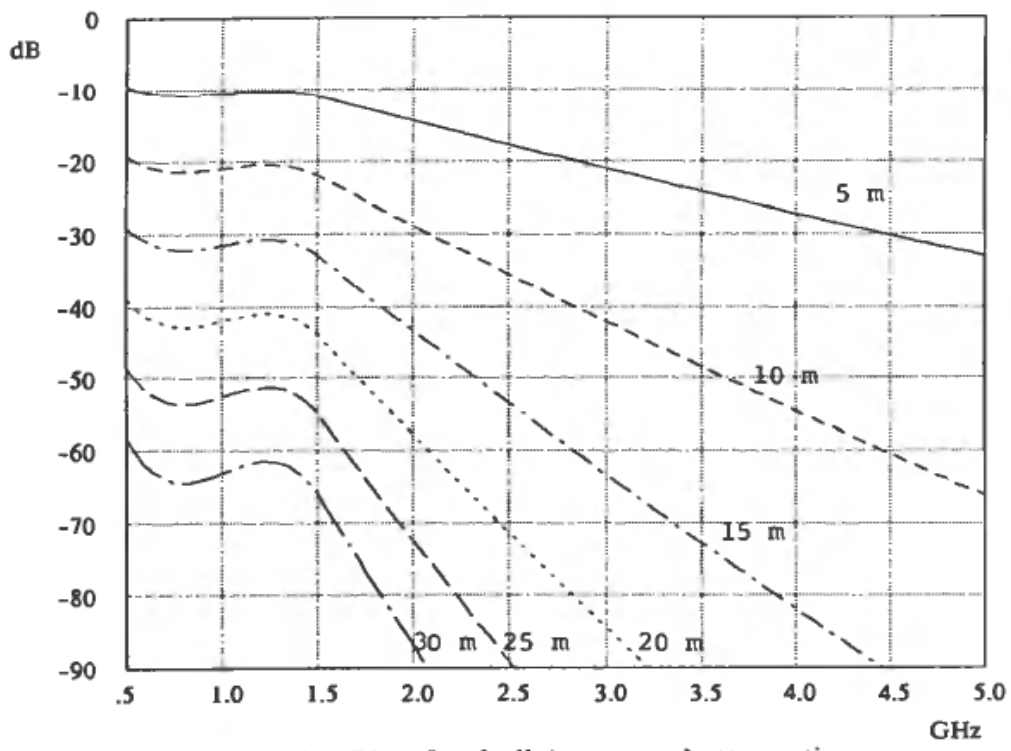

Figure 11. Unrefined oil, 'two-way' attenuation.

referred to using the following number:

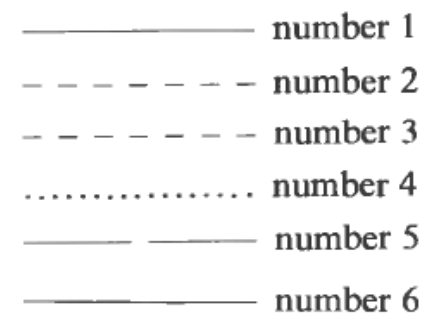


Figure 12 shows the dielectric constant and the attenuation in pure water $(S=0)$. At small frequencies the real part has a flat area and the value sinks from about 88 to 72. The imaginary part has relaxation absorption (von Hippel, 1952) which varies from approximately 9 to $30 \mathrm{GHz}$.

This leads to an attenuation which is shown in Fig. 12(c). If we assume that we can use $30 \mathrm{~dB}$ to 'look through' the water, then we can detect the bottom under $15 \mathrm{~cm}$ of water by $1.5 \mathrm{GHz}(T=20)$. In a small separation tank we can probably use $50-60 \mathrm{~dB}$. This will allow depths of about $25 \mathrm{~cm}$ to be detected. However, at this stage it should be pointed out that we do not need to detect the bottom under

a)

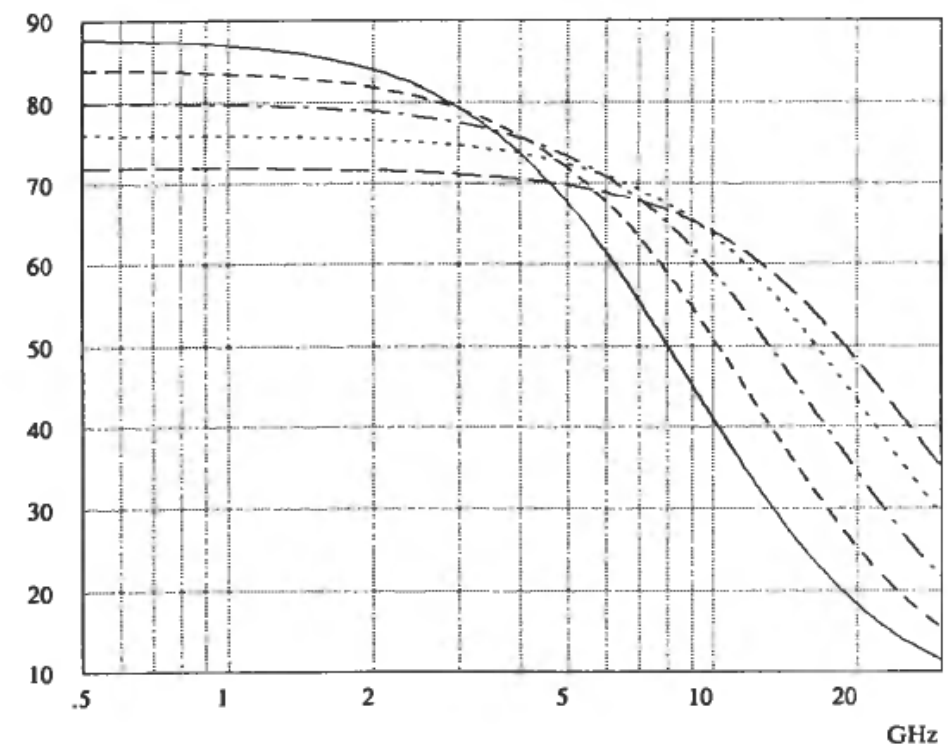

b)

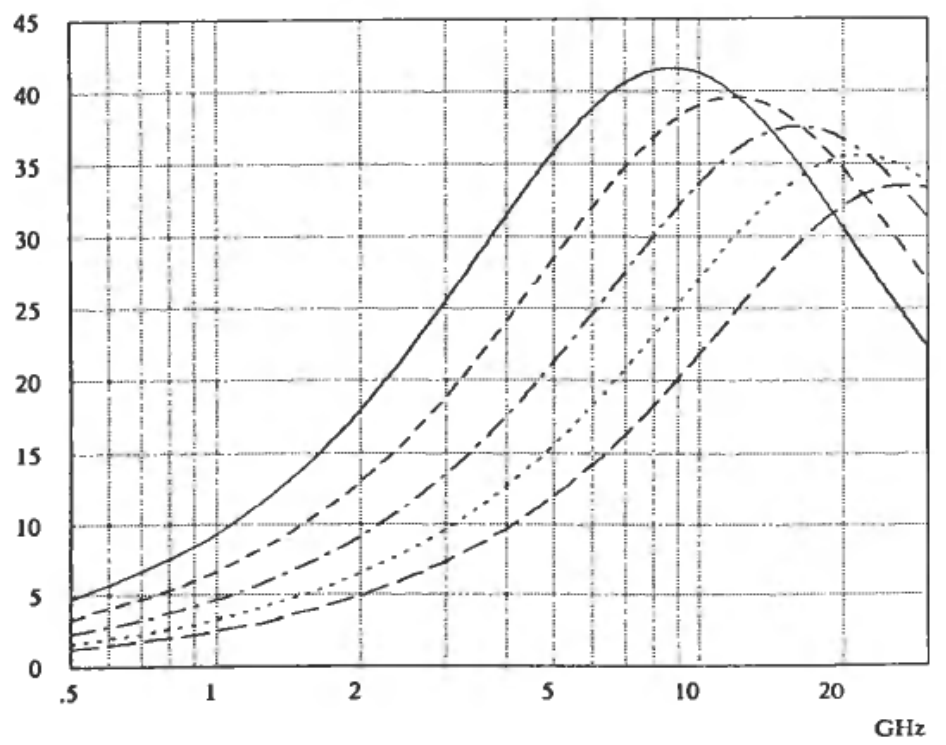

Figure 12. Pure water. (a) $\varepsilon^{\prime} / \varepsilon_{0} . T=0,10,20,30,40$; (b) $\varepsilon^{\prime \prime} / \varepsilon_{0} . T=0,10,20,30,40$; (c) 'Two-way' attenuation by $1.5 \mathrm{GHz} . \mathrm{T}=0,10,20,30,40$. 


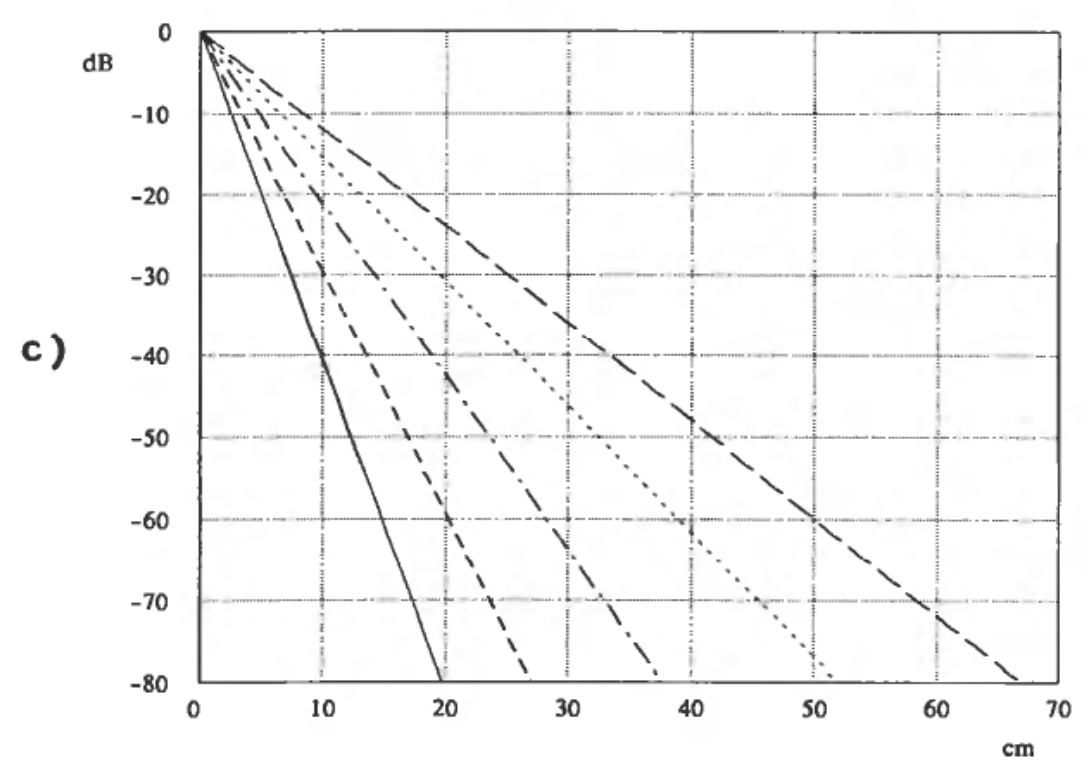

the water in order to know the depths of the oil and water. If we can determine the impedance transition between the oil and the water, then we are able to calculate the depth of the water if the depth of the tank is known.

5.2.2. Sea water Figure 13 shows the dielectric constant and the attenuation in sea water $(S=35)$. The shape of the relative real part of the dielectric constant of sea water is much the same as the shape in Fig. 12(a). The only difference is the lowering of the values at small frequencies.

a)

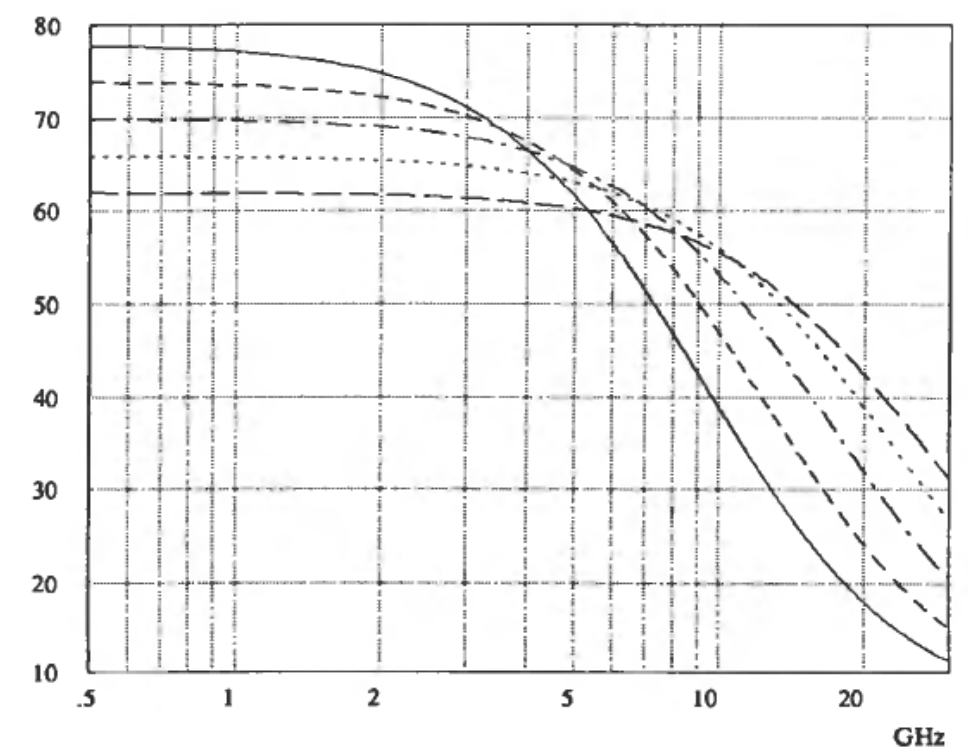

Figure 13. Sea water. $(a) \varepsilon^{\prime} / \varepsilon_{0} . T=0,10,20,30,40 ;(b) \varepsilon^{\prime \prime} / \varepsilon_{0} . T=0,10,20,30,40$; (c) "Twoway' attenuation by $1.5 \mathrm{GHz} . T=0,10,20,30,40$. 

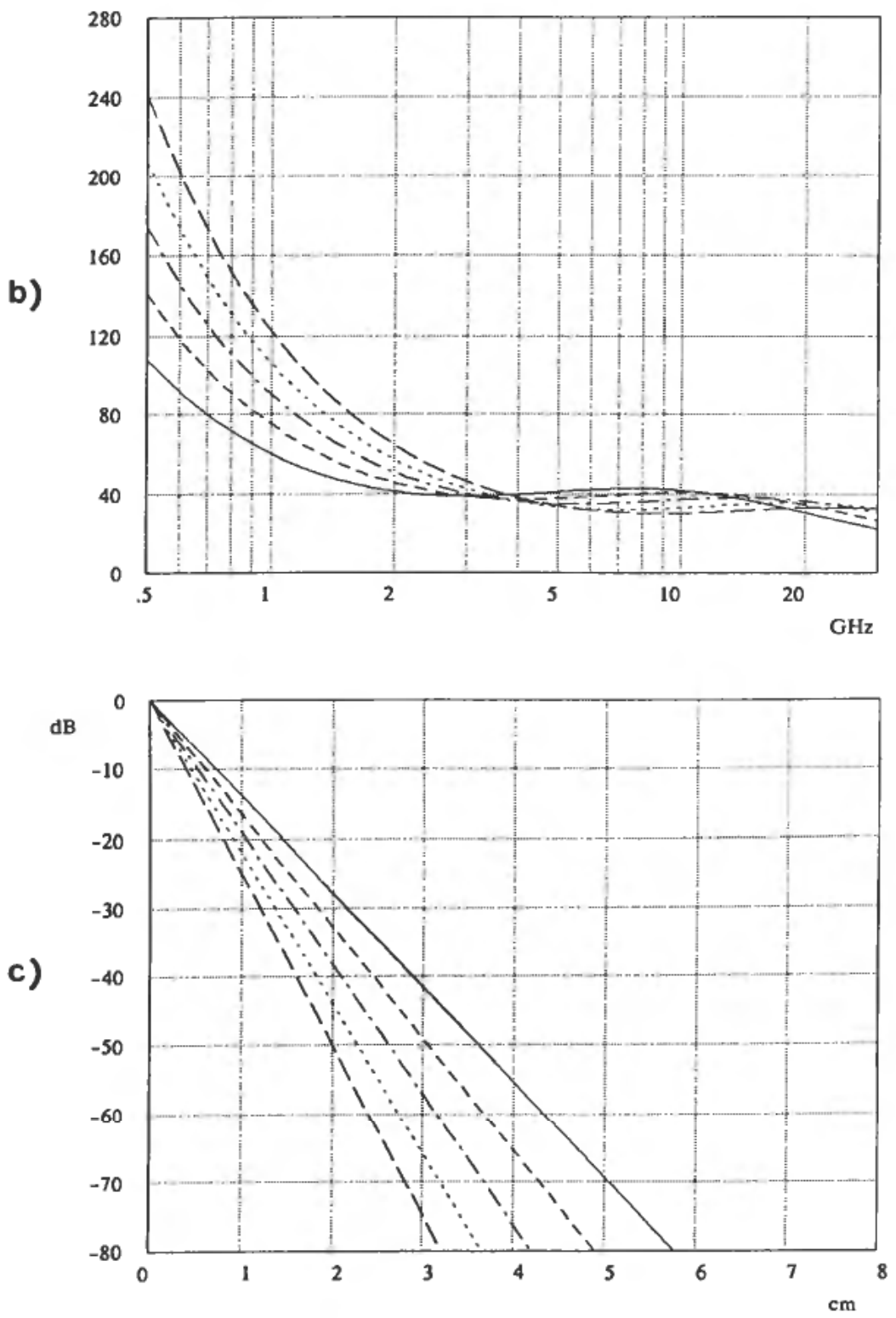

However, for the imaginary part the change is dramatic. The reason for this is the influence of the ion content caused by the $\mathrm{NaCl}$. The effect of the relaxation absorption and the ions is that $\varepsilon^{\prime \prime}$ in the range from 3.5 to $20 \mathrm{GHz}$ is relatively independent of the temperature.

Figure 13(c) shows the 'two-way' attenuation. The principal influence of the temperature is not the same as for pure water. In addition, the level of the attenuation has now been changed dramatically. In a small separation tank (att. 50-60 dB, $20^{\circ} \mathrm{C}$ ) we are only capable of 'seeing through' layers of about $3 \mathrm{~cm}$. However, as mentioned earlier, we do not need to detect the bottom in order to calculate the depths of the oil and water. 


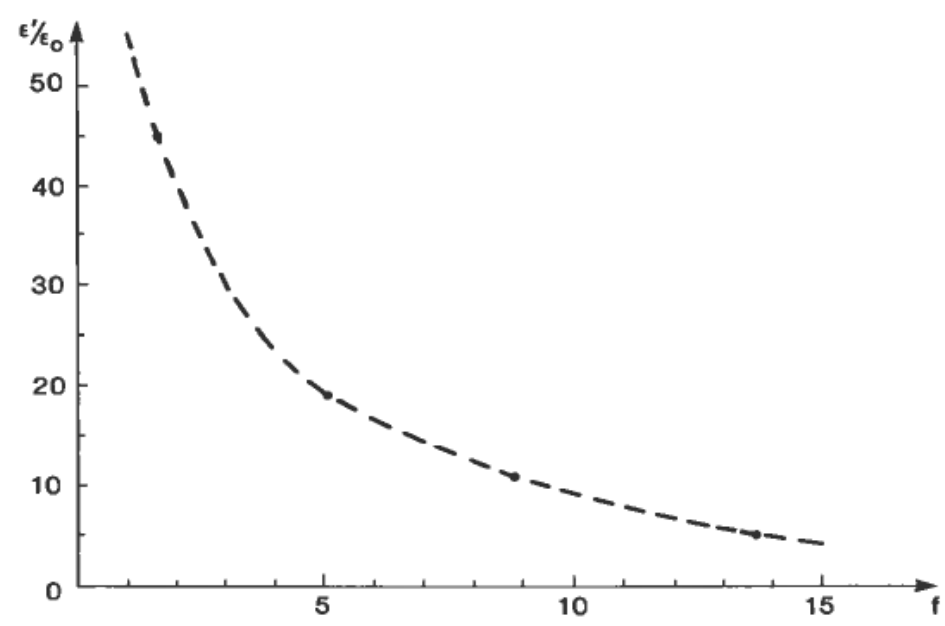

Figure 14. Waxed oil (mud), mean value of $\varepsilon^{\prime} / \varepsilon_{0}$.

\subsection{Waxed oil (mud)}

Figures 14 and 15 show the dielectric constant of the waxed oil (mud). The relative real part drops smoothly from about 50 to 5 , while the imaginary part falls from approximately 6 to 1 . The weekly rise at higher frequencies is probably caused by the water content in the waxed oil.

Figure 16 illustrates the attenuation. At a frequency of $1.5 \mathrm{GHz}$ we are capable of 'looking through' a layer of waxed oil of about $25 \mathrm{~cm}$ in small separation tanks (no water).

\section{Simulations}

As pointed out in $\S 3$, the expressions which describe the tank quickly became rather complicated, and in this section different situations of interest are simulated on a computer in order to simplify the analyses. The calculations are based on the dielectrical data which are presented in $\S 5$. So far the losses and possible ranges in

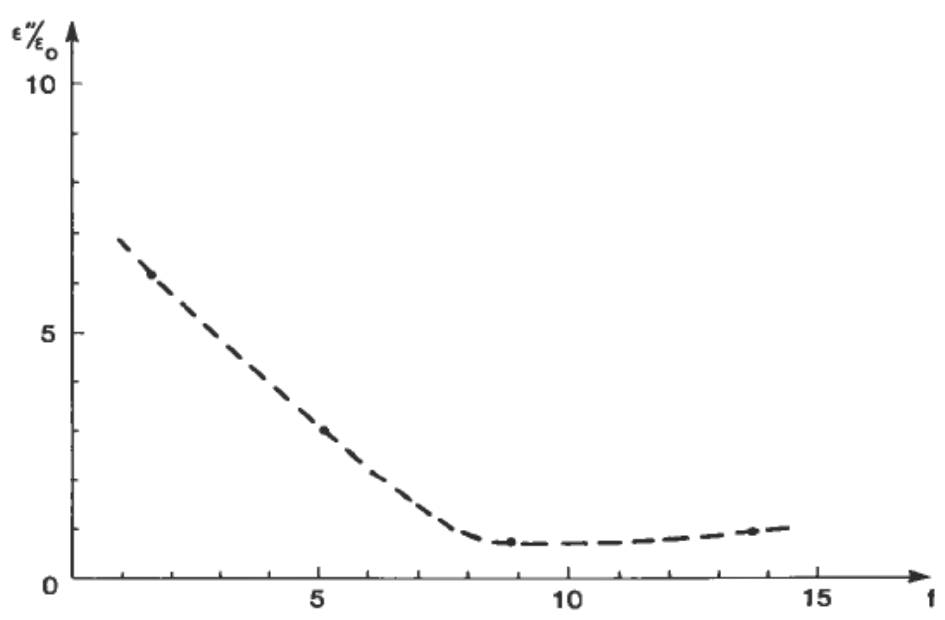

Figure 15. Waxed oil (mud), mean value of $\varepsilon^{\prime \prime} / \varepsilon_{0}$. 


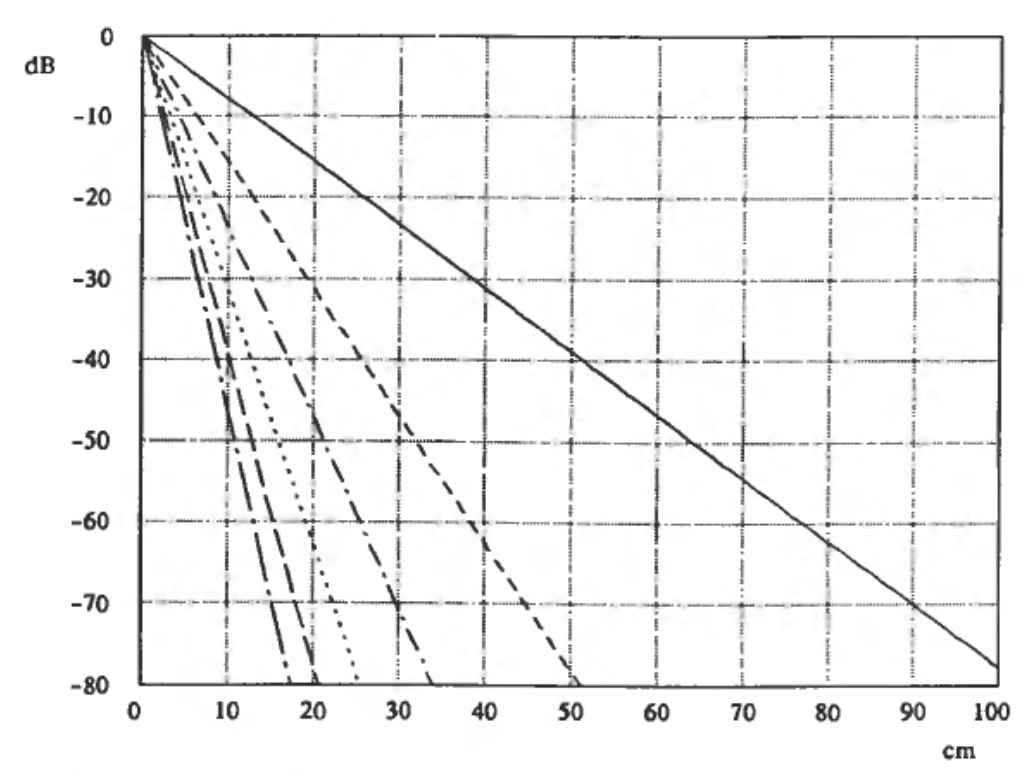

Figure 16. Waxed oil (mud), 'two-way' attenuation. $F=0 \cdot 5,1 \cdot 0,1 \cdot 5,2 \cdot 0,2 \cdot 5,3 \cdot 0$.

the different layers have been analysed. In $\S 6$ methods of detection and the use of the windows which were presented in $\S 4$ are discussed.

\subsection{Detection of water}

First a oil-tank with a height of $5.2 \mathrm{~m}$ is simulated. At the bottom there is a layer of pure water which is $20 \mathrm{~cm}$ deep. The frequency band is from $1.1-1.9 \mathrm{GHz}$ and the number of frequencies is 64 . The simulations are carried out at a temperature of $15^{\circ} \mathrm{C}$ in the water, see Fig. $17(a)$. Here Rectangular, Hamming and Blackman windows are used. The impedance transition between the oil and water is at $5 \mathrm{~m}$. The relative dielectric constant in the oil is approximately $2 \cdot 15$.

The electrical distance from the surface to the water becomes:

$$
Z_{w} \simeq \sqrt{ } 2.15 \times 5 \mathrm{~m} \simeq 7.33 \mathrm{~m}
$$

Here, the relative dielectric constant in the water is about 82 . The echo from the bottom then should appear at the following range:

$$
Z_{b 1} \simeq Z_{w}+\sqrt{ } 82 \times 20 \mathrm{~cm} \simeq 9 \cdot 14 \mathrm{~m}
$$

With the use of the Rectangular window we can detect the surface of the water, but the bottom is masked by the side lobes. However, knowing the surface of the oil, the height of the tank and the dielectric constant in the oil we can easily calculate the depth of the water:

$$
Z_{d^{\prime}} \simeq 5 \cdot 2 \mathrm{~m}-\frac{Z_{w}}{\sqrt{2 \cdot 15}}
$$

With the use of the Hamming window we can still not see the bottom, but a dip at about $9.15 \mathrm{~m}$ is observed. This indicates that an echo of approximately equal strength to the side lobes and in opposite phase is situated at this range. The Black- 

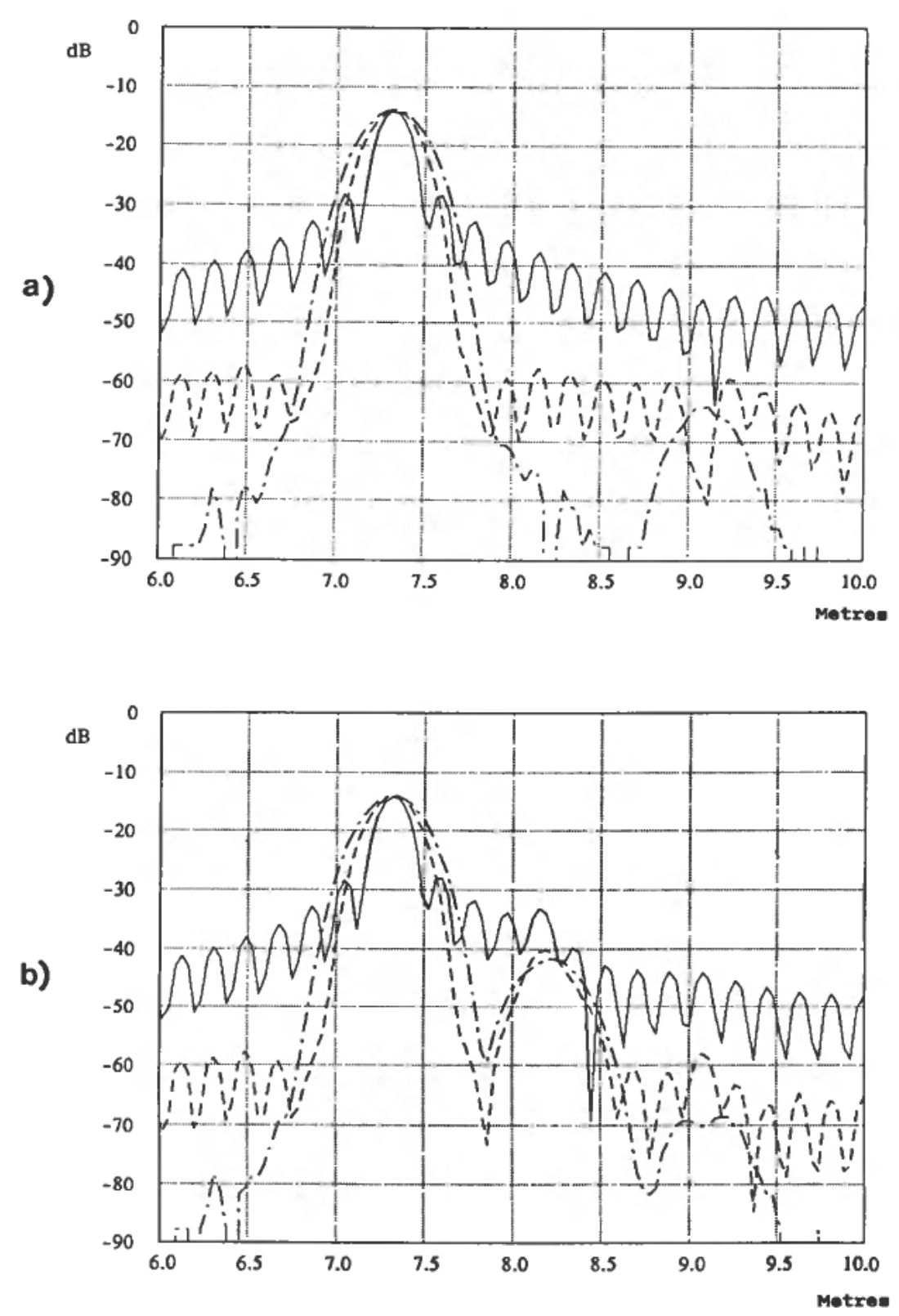

Figure 17. Detection of water. (a) Depth $20 \mathrm{~cm}$. Rectangular, Hamming, Blackman; (b) Depth $10 \mathrm{~cm}$. Rectangular, Hamming, Blackman.

man window has such small side lobes that the bottom can be detected directly. Eqn (18) can now be checked:

$$
Z_{d^{\prime \prime}} \simeq \frac{Z_{b 1}-Z_{w}}{\sqrt{82}}
$$

Figure 17(b) shows the situation with a height of $5.1 \mathrm{~m}$. The layer of water is now $10 \mathrm{~cm}$. The Rectangular window still only gives the surface of the water. However, 
the attenuation in the water is now so low that the Hamming window can be used. The bottom is situated at:

$$
Z_{b 2} \simeq Z_{w}+\sqrt{ } 82 \times 10 \mathrm{~cm} \simeq 8.24
$$

The distances in Eqns (16), (17) and (20) agree very well with the simulations in Fig. 17. Using the Blackman window in Fig. 17(b) we can even detect the first multiple echo from the bottom.

\subsection{Detection of waxed oil (mud)}

The complexity is now expanded by simulating a layer of waxed oil (mud) at the bottom in addition to the water. The frequency band is still $1.1-1.9 \mathrm{GHz}$ with 64 frequencies. The temperature is $15^{\circ} \mathrm{C}$ and the layer of oil $4 \mathrm{~m}$.

Figure 18(a) shows the response with a layer of water of $15 \mathrm{~cm}$ and waxed oil of $7.5 \mathrm{~cm}$. The weighting functions used are the Hanning and the Hammingfolded windows. With the Hanning window we see that the side lobes arc overlapping the bottom and waxed oil echo, but such problems are not present with the Hammingfolded window. Here the echoes are clearly visible from the water surface, the waxed oil and the bottom. It is obvious that the bottom echo is not a multiple of the waxed oil echo. The distances between the three echoes are not the same and the level of the last echo is higher than the waxed oil echo.

In Fig. 18(b) the layer of water is $7 \mathrm{~cm}$ and the layer of waxed oil $4 \mathrm{~cm}$. With the Hanning and the Hammingfolded windows we can see the water echo and the bottom echo. In addition, one multiple bottom echo is visible. The echo from the waxed oil is masked by either the main echoes or the side lobes.

Figure 18(c) shows the result of a new advanced method of processing signals (described in a forthcoming paper by the present author). We see the processed

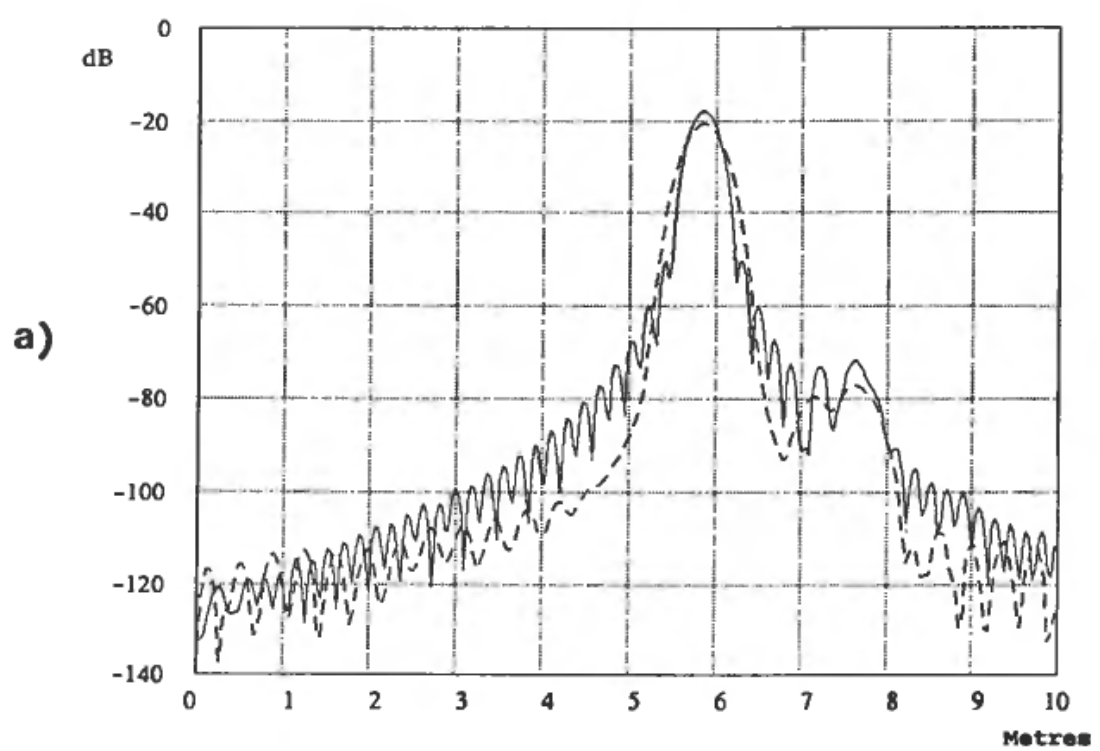

Figure 18. Detection of waxed oil (mud). (a) Oil $4 \mathrm{~m}$, water $15 \mathrm{~cm}$, waxed oil $7.5 \mathrm{~cm}$, Hanning, Hammingfolded; (b) Oil $4 \mathrm{~m}$, water $7 \mathrm{~cm}$, waxed oil $4 \mathrm{~cm}$, Hamming, Hanning, Hammingfolded; (c) Processed data, Processed bottom echo, synthetical echo, processed waxed oil echo. 

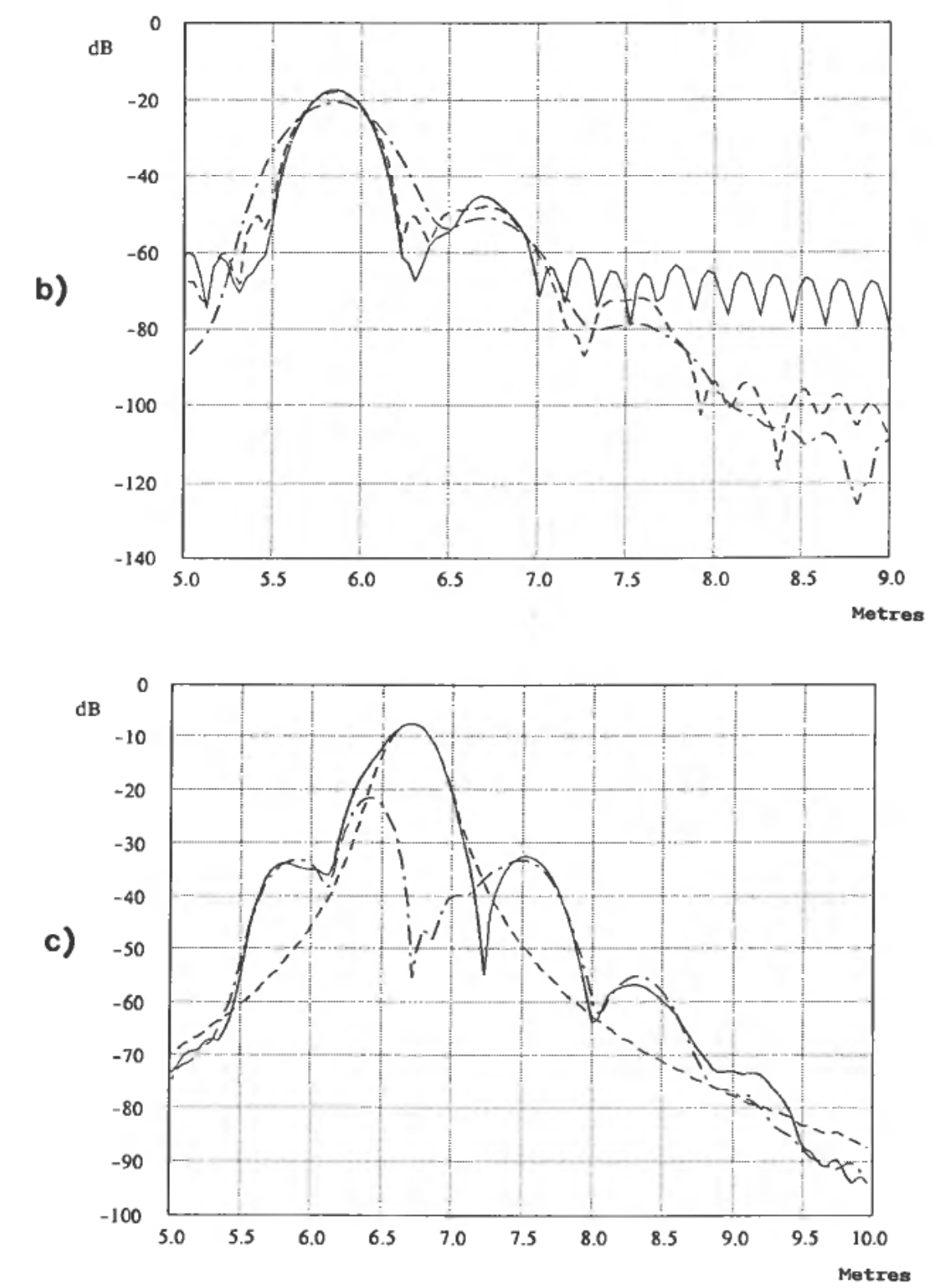

bottom echo, a synthetical process echo and the processed waxed oil echo. The waxed oil echo should appear at the following range:

$$
Z_{w_{0}} \simeq \sqrt{ } 2.15 \times 4 \mathrm{~m}+\sqrt{ } 82 \times 7 \simeq 6.5 \mathrm{~m}
$$

As Figure 18(c) shows this is approximately correct.

\subsection{Detection of emulsion}

6.3.1. Description of the model The layer of emulsion is a volume between the water and the pure oil where waterdrops are fleeting in the oil, see Fig. 19. The drops are touching the surface of the water, and can here fill approximately $70 \%$ of 


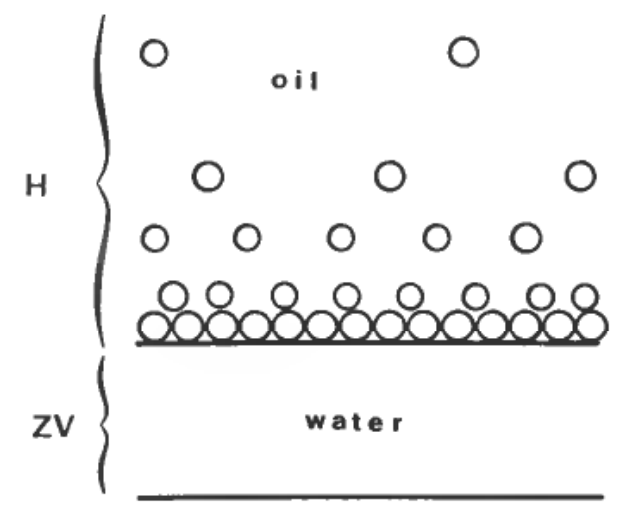

Figure 19. Emulsion.

the volume. They are extended far up in the oil, but in the simulations it is supposed that they become electrically significant at a distance $H$ from the surface of the water. The profile is described by the following equation (Fig. 20):

$$
F X=W\left(\frac{H+Z 1+Z V-Z}{H}\right)^{Q}
$$

The reflection coefficient is calculated supposing one thousand impedance transitions through the layer of emulsion.

6.3.2. Results from the simulations First the necessary degree of weighting is explored. The simulations are carried out with only a layer of water under the oil. The frequency band is from $1 \cdot 1-1.9 \mathrm{GHz}$ and the number of frequencies 32 . The oil is $1 \mathrm{~m}$ and the water $15 \mathrm{~cm}$. The temperature is $15^{\circ} \mathrm{C}$ and only pure water is used. Figure 21 shows the response with Rectangular, Hanning and Hammingfolded windows. The side lobes create problems even with the Hanning window, and in the further simulations of emulsion only the Hammingfolded window is used. The figure first shows the surface of the water and after that the bottom. In addition, a weekly

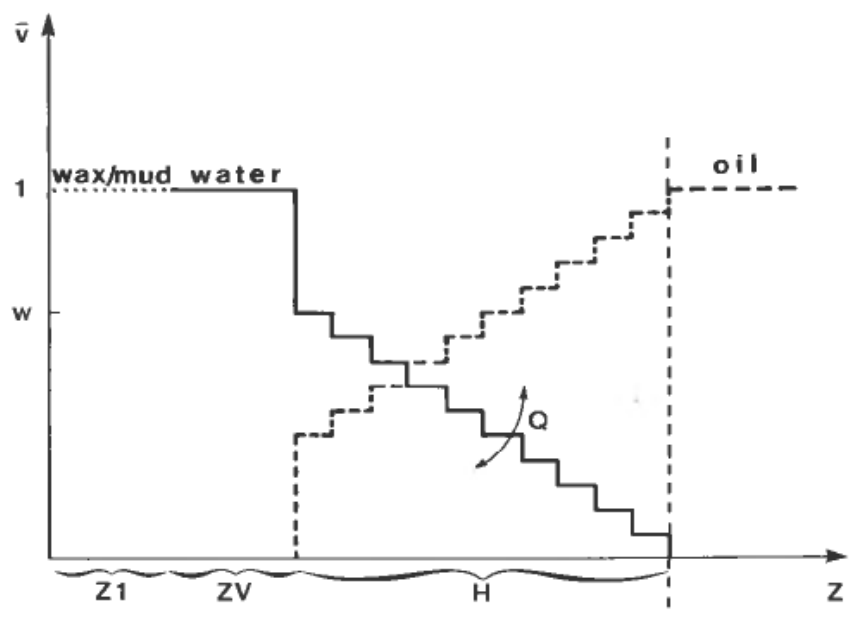

Figure 20. Calculation model. 


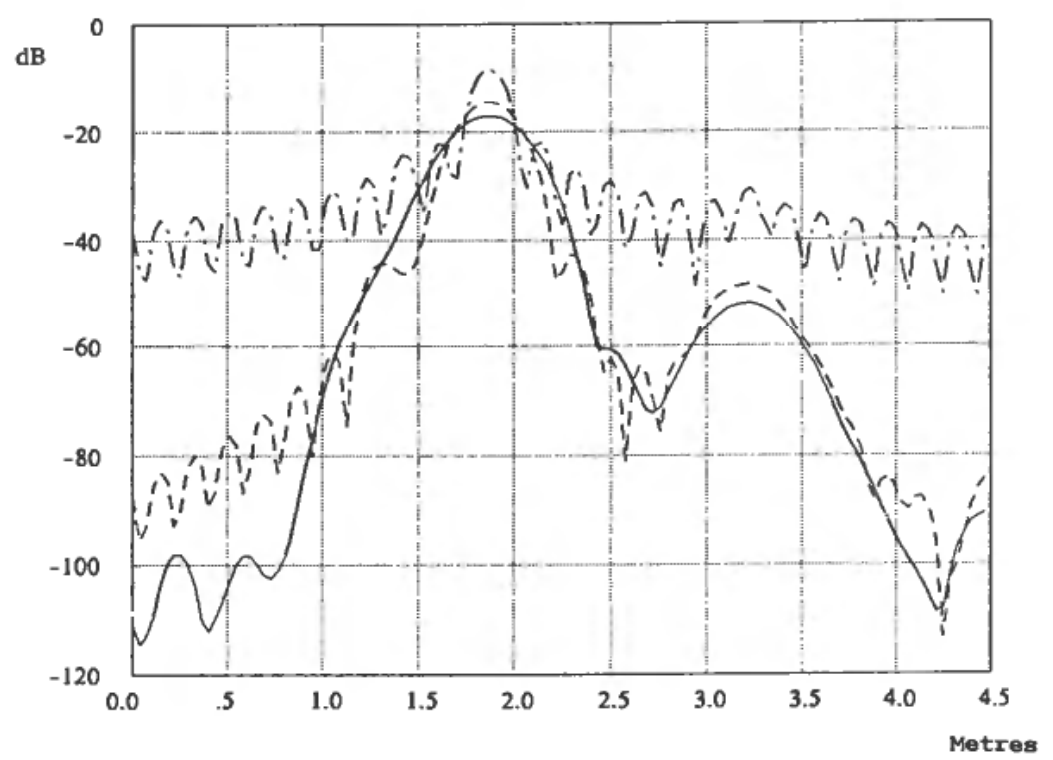

Figure 21. Use of windows. $H=20 \mathrm{~cm}, W=0.7, Q=1$. Rectangular, Hanning, Hammingfolded.

rise of the levels around $1.1 \mathrm{~m}$ and $2.5 \mathrm{~m}$ is observed. The signal at $1.1 \mathrm{~m}$ is an echo from the first impedance transitions where the waterdrops become electrically significant, and the signal at $2.5 \mathrm{~m}$ is the first multiple echo from these transitions. We can get further information about the start of the emulsion by either using a wider frequency band or applying the new advanced methods of processing signals (mentioned earlier).

In Fig. $22(a)$ the parameter $W$ is varied from 0.4 to $1 \cdot 0$. The frequencies are the same as in Fig. 21. Four effects can be noticed in particular. Firstly, the echo from the emulsion rises with rising $W$. This can be explained by the increased impedance transitions at the start of the emulsion. Secondly, the echo from the water is slightly reduced with rising $W$. This effect is caused by the increased reflection from the emulsion. More detailed studies, which are not referred to here, show that the attenuation in the drops is of secondary importance. Thirdly, the distance to the water echo is increasing with rising $W$. The reason for this is that the bigger volume of waterdrops increases the effective dielectric constant. The degree of movement is a function of the number of waterdrops, and can be used to estimate the volume of the waterdrops. And finally, the echo from the bottom has disappeared. This is caused by the salt content which is $15 \mathrm{~g} / \mathrm{l}$.

Figure 22(b) shows the effect of varying the extent of the emulsion layer. The frequencies are the same as in Fig. 21. The principal effects are equal to those in Fig. 22(a). The only difference is that the rise in the emulsion echo is caused by the reduced distance to the emulsion layer. The losses in the oil are then smaller.

To analyse the effect of increasing $Q$ it is convenient to choose a bandwidth which makes it possible to distinguish the water and the emulsion echo directly. In Fig. 22(c) the frequency band is from $1-5 \mathrm{GHz}$ with 128 frequencies. The echo from the emulsion is now clearly visible. The usual movement in the water echo with the rising number of waterdrops is also observed. One very interesting effect is that the 
a)
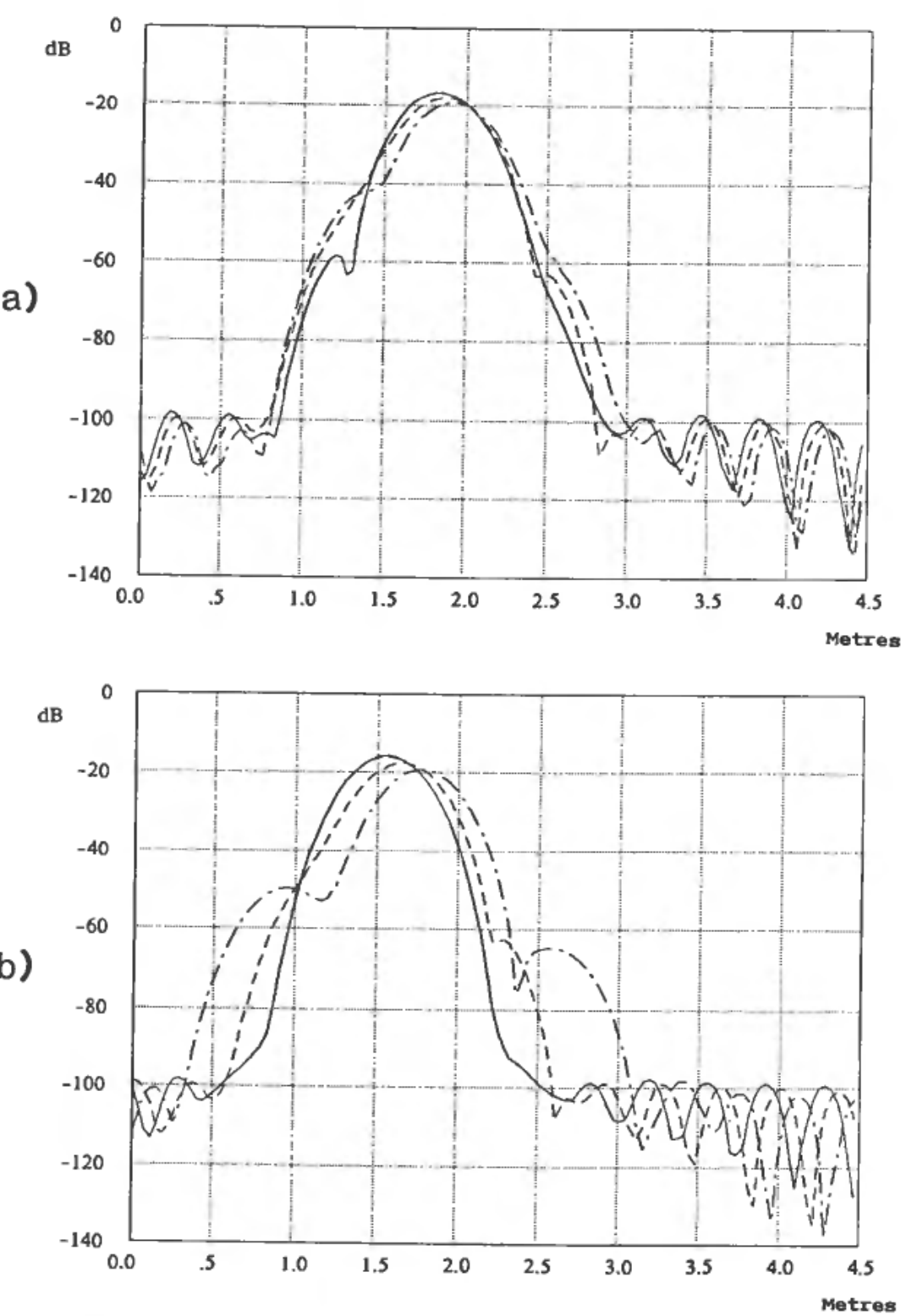

Figure 22. Variation of emulsion profile. (a) Oil $1 \mathrm{~m}$, water $15 \mathrm{~cm}, H=20 \mathrm{~cm}, Q=1$, $T=15, S=15, W=0.4,0.7,1 \cdot 0 ;(b)$ Oil $+H=1.05 \mathrm{~m}$, water $15 \mathrm{~cm}, W=0.7$, $Q=1, T=15, S=15, H=5 \mathrm{~cm}, 20 \mathrm{~cm}, 40 \mathrm{~cm}$; (c) Oil $1 \mathrm{~m}$, water $15 \mathrm{~cm}, H=15 \mathrm{~cm}$, $W=0 \cdot 7, T=15, S=15, Q=1,2,5$.

emulsion echo seems to be almost independent of $Q(Q \geqslant 2)$. As soon as some waterdrops are present we obtain a distinct echo which marks the start of the emulsion layer.

Detection of the start of the emulsion can be carried out in several ways. If the frequency bandwidth and the dynamic range are large enough, we can detect the start directly as shown in Fig. 22(c). Alternatively we can use the new method of processing signals. And finally, if we know the surface of the water and the dielectric constant in the oil we can calculate the start of the emulsion indirectly. 


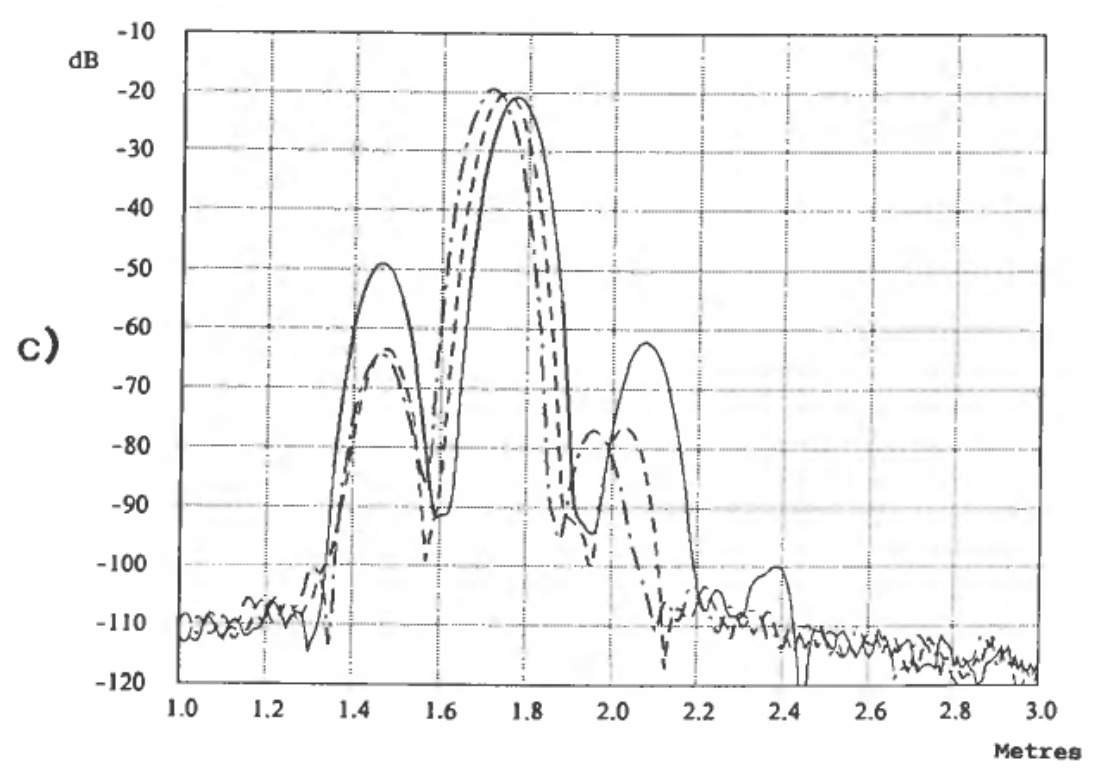

If we suppose that $W=0.7$ and know the start of the emulsion, then the effective volume of the waterdrops and the profile of the emulsion be calculated.

\subsection{Detection of foam}

Up to now the situation at the bottom of the tank has been simulated and analysed. In this section, possible responses from foam and the surface of the oil are discussed.

The nature of the foam is illustrated in Fig. 23. The smallest bubbles are situated at the surface. The diameter of the bubbles increase with increased distance from the surface. This effect will create softly dielectrical changes between the surface of the foam and the oil. However, the surface of the foam will represent a sharp dielectrical transition and cause a distinct echo. The same will also happen at the surface of the oil, but here small bubbles of gas may be situated in the oil close to the surface. The density of bubbles in the oil will probably increase with an increasing layer of foam. This effect may create a diffuse impedance transition and reduce the echo from the surface of the oil.

Figure 24 illustrates possible responses from the surface. No foam results in a clear surface echo. A small layer of foam will raise the level to the left of the top.

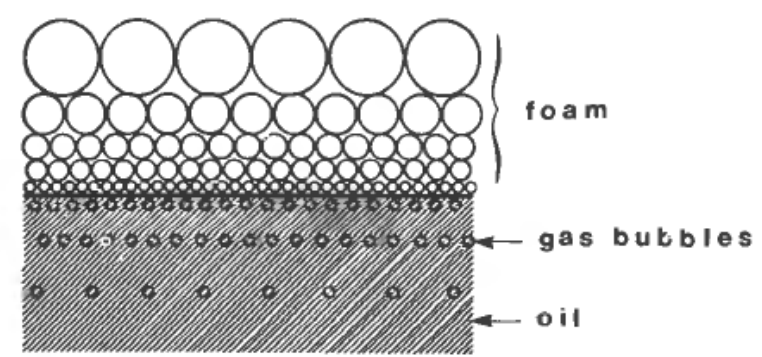

Figure 23. Model of foam. 


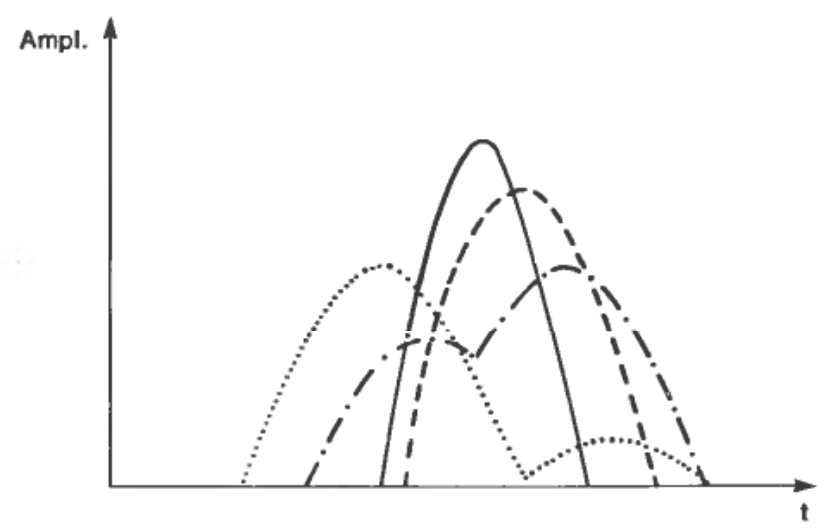

Figure 24. Response from the surface; No foam, little foam, medium foam, much foam.

With the new method of processing signals information about the start of the foam layer can be extracted from the data. A medium layer of foam may give a distinct foam echo, and if the layer is increased further the echo from the foam may be the strongest. As the foam layer increases, the echo from the oil surface will move away from the reference plan. This can be explained by the dielectric constant of the foam. Advanced signal processing methods can be used if the echo from the oil surface is strongly reduced. The surface of the foam or the oil can also be calculated indirectly as in the case with emulsion and water.

The problems with the reduced echo from the oil surface is especially important at higher frequencies (freq. $>7-8 \mathrm{GHz}$ ). This effect will probably not create problems at the frequencies which are used in these simulations.

\section{Practical measurements}

\subsection{Description of the practical tank simulation}

In order to verify parts of the theoretical simulations the tank is simulated by a coaxial line, see Fig. 25. It simply consists of a circular pipe with a centre conductor. Theoretically, a coaxial line can conduct frequencies from zero up to infinity. However, problems will arise when we are approaching the cut-off for the pipe (about $5 \mathrm{GHz}$ ). The practical measurements are carried out with an automatic network analyser and the same frequency band as in the simulations $(1 \cdot 1-1.9 \mathrm{GHz})$ is used. The number of frequencies is 401 . The reflection coefficient is measured at the top of the feed, and both the practical connection and the end of the epoxy layer will create echoes. However, these echoes can simulate the reflections from the antenna. At the bottom of the pipe different kinds of reflectors can simulate water and waxed oil or foam and the surface of the oil.

\subsection{Measurements with refiectors}

Figure 26 shows some measurements using reflectors. First the response of empty pipe is measured. We see the echo from the epoxy at about $0.25 \mathrm{~m}$ and the echo from the bottom at approximately $1.45 \mathrm{~m}$. The echo from the reference plan is masked by the echo from the epoxy, but can be extracted by further signal processing. 


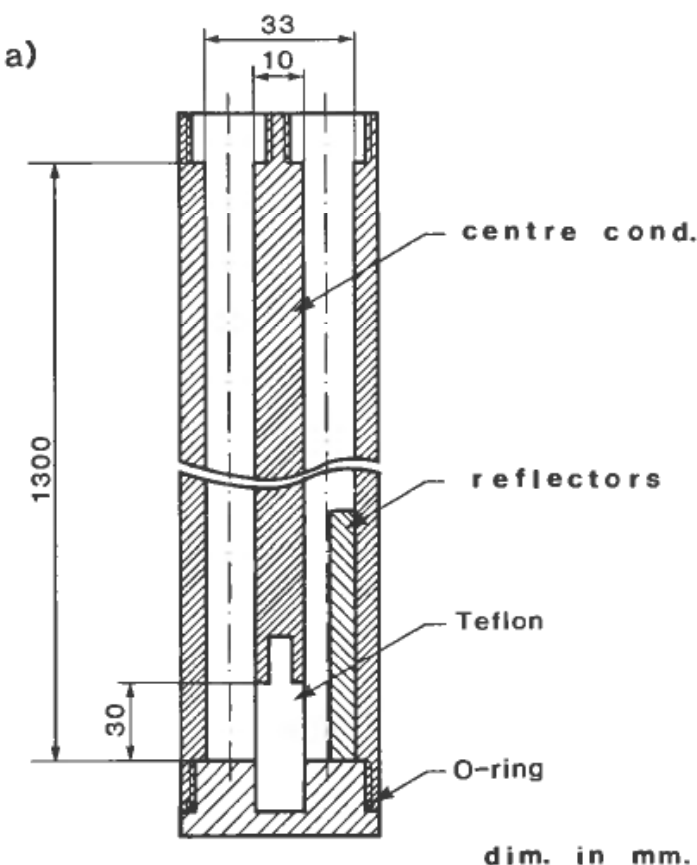

b)

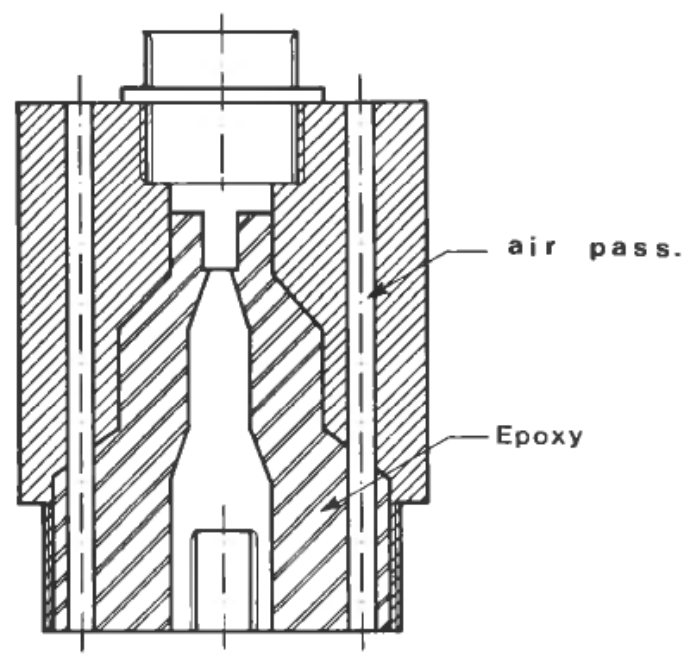

Figure 25. Practical tank simulation. (a) Coaxial line, (b) Feed.

In Fig. $26(b)$ the reflector is $50.5 \mathrm{~cm}$. This range is outside the main lobe if the Hanning window is used $(37.5 \mathrm{~cm})$, but inside the main lobe if the Hammingfolded window is used instead $(75 \mathrm{~cm})$. The figure shows that the reflector echo is situated at about $1 \mathrm{~m}$ with a multiple echo at about $2 \mathrm{~m}$. The bottom is at $1.45 \mathrm{~m}$ with a multiple bottom echo at approximately $2 \cdot 7 \mathrm{~m}$.

In Fig. $26(c)$ the reflector is reduced to $23 \mathrm{~cm}$. It is situated inside the main lobe, but further signal processing extracts the required information from the measured data. The reflector echo is visible at about $1.2 \mathrm{~m}$ with multiple echoes after the bottom echo. The practical measurements in this section clearly demonstrate that it 
is possible to identify both large and small impedance transitions by means of the reflectometer principle.

\subsection{Measurements with oil and water}

In this section the practical experiments are expanded to consider real measurements with oil and water. Figure 27(a) shows the response with only oil in the pipe. If we compare this with Fig. 26(a) we will notice three main differences. Firstly, the bottom has moved from $1.45 \mathrm{~m}$ to approximately $2.05 \mathrm{~m}$. This effect is caused by
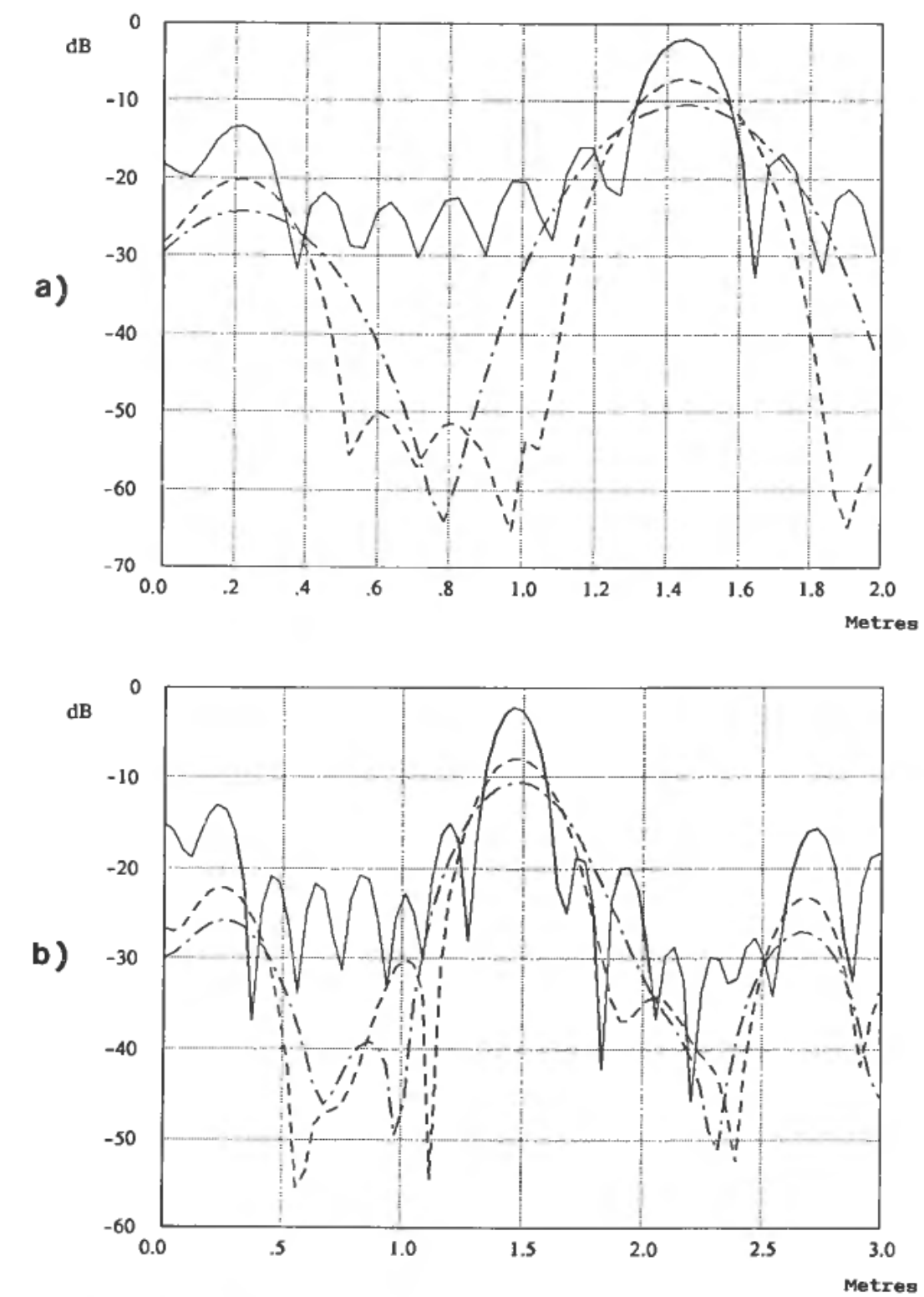

Figure 26. Measurements with reflectors. (a) Empty pipe. Rectangular, Hamming, Hammingfolded; (b) Reflector 50.5 cm. Rectangular, Hanning, Hammingfolded; (c) Reflector $23 \mathrm{~cm}$. Measured echo, synthetical echo, processed reflector echo. 


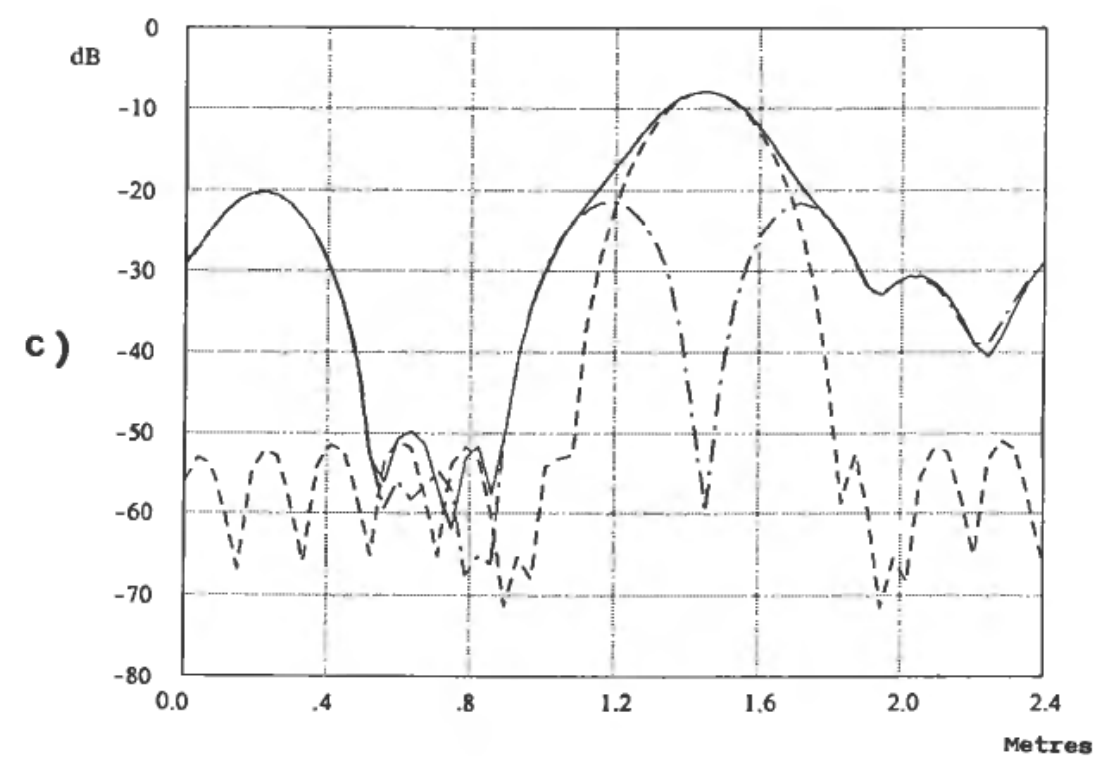

the dielectric constant in the oil. Secondly, the level of the bottom echo is reduced by about $3 \mathrm{~dB}$. This reduction can be explained by the attenuation in the oil. The changed impedance transition between the epoxy and the oil is only of secondary importance. But the third effect, which is the reduced epoxy echo, is caused by this change of impedance transition. The echo in the reference plan is now visible without further signal processing.

In Fig. 27(b) part of the pipe is filled with $14 \mathrm{~cm}$ of fresh water. It is now very important to remember that the part of the pipe which is filled with water is oper-

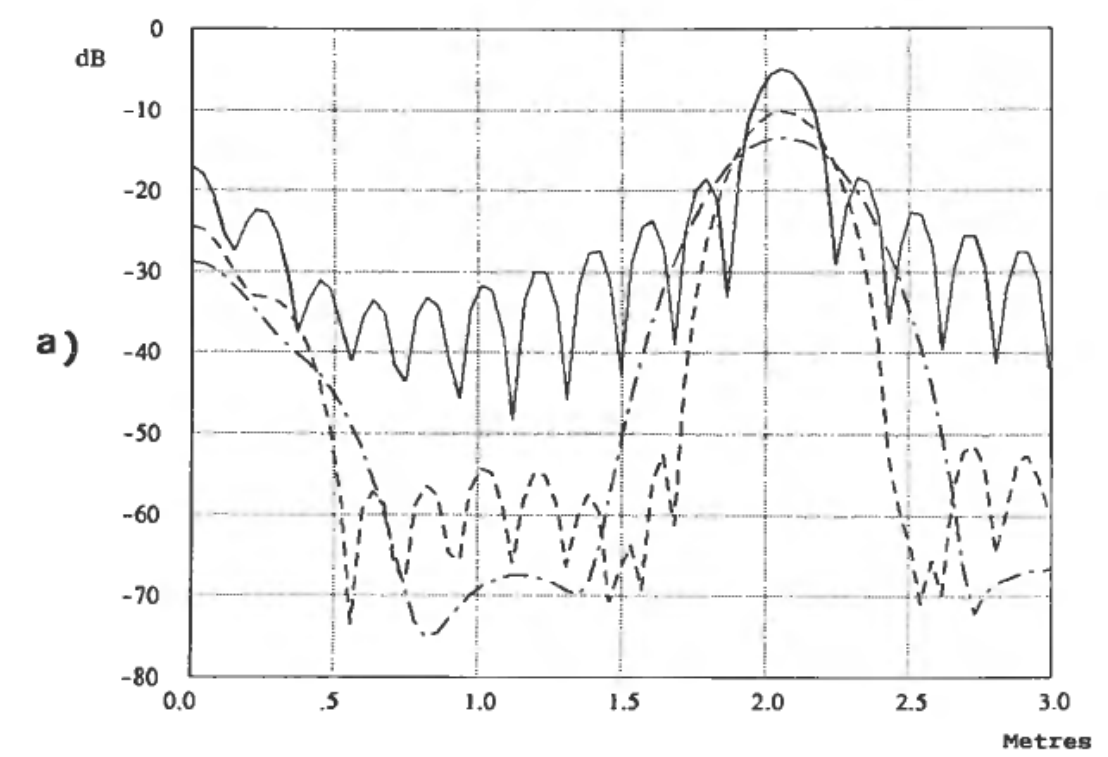

Figure 27. Measurements with oil and water. (a) No water. Rectangular, Hamming, Hammingfolded; (b) Water $14 \mathrm{~cm}$. Hamming, Hanning, Hammingfolded; (c) Water $5 \mathrm{~cm}$. Hamming, Hanning, Hammingfolded. 

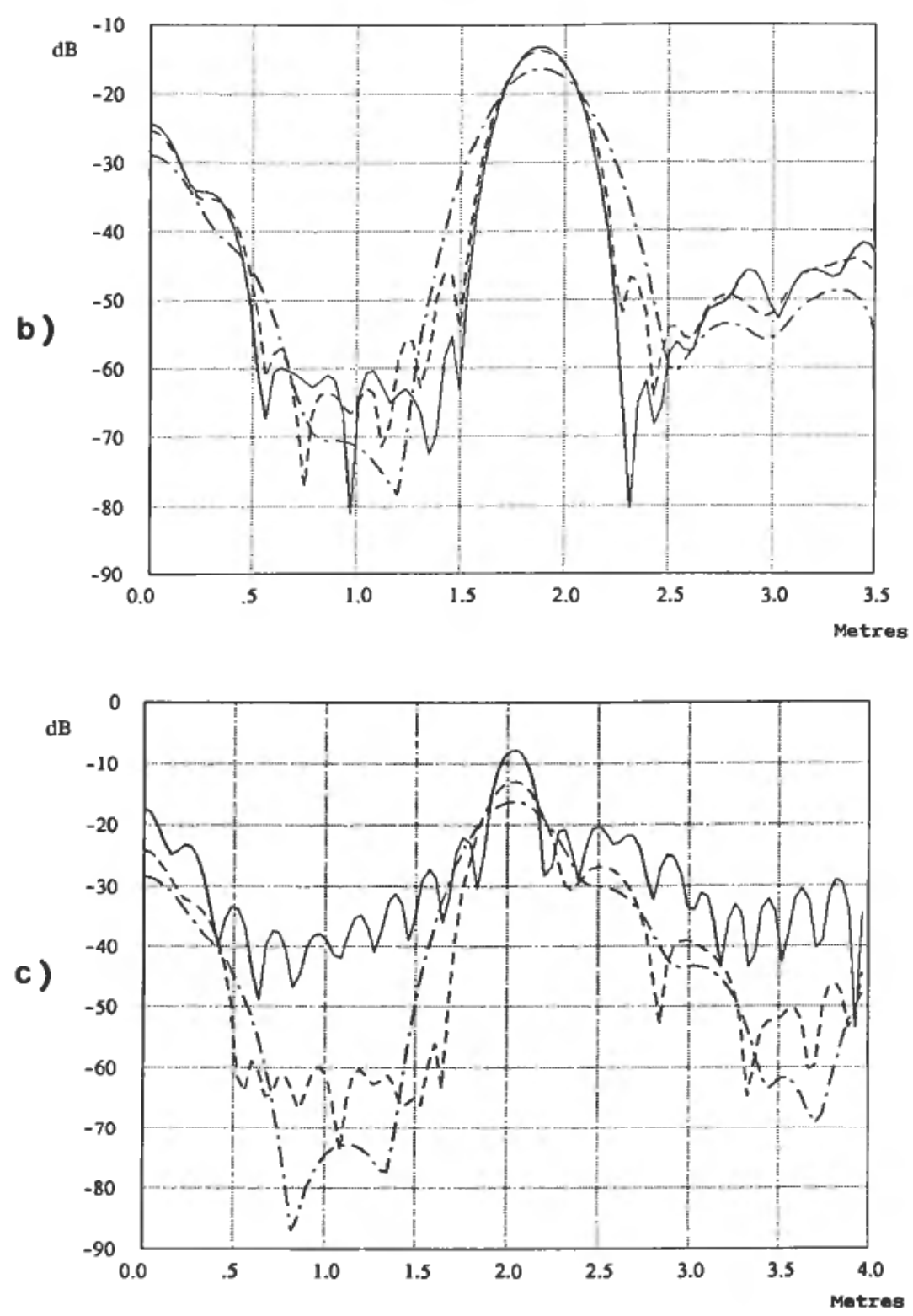

ating over the cut-off. The reason for this is the high dielectric constant of the water, which means that the bottom ought to be detected under the end of the centre conductor. With the Hammingfolded window the figure shows the surface of the water at about $1.85 \mathrm{~m}$, the end of the centre conductor at $2.80 \mathrm{~m}$ and the bottom at $3.30 \mathrm{~m}$. This figure demonstrates that we are capable of identifying the reference plan, the end of the epoxy layer, the water surface, the end of the centre conductor and the bottom in practical measurements.

Figure $27(c)$ shows the response with a layer of water of $5 \mathrm{~cm}$. We see the water, the bottom and two multiple bottom echoes. The end of the centre conductor is now masked by the water and bottom echo, but can be extracted by further signal processing. 


\section{Conclusion}

The simulations clearly demonstrate that it is possible to identify practical layers in oil-tanks by means of the reflectometer principle. In small separation tanks it may be possible to 'look through' $7-8 \mathrm{~cm}$ of water or about $25 \mathrm{~cm}$ of waxed oil (mud). If we are not capable of 'looking through' the different layers directly, it may be possible to calculate the impedance transitions indirectly. Both foam and the surface of the oil should be able to be detected directly or indirectly.

The practical measurements verify that the reflectometer principle is applicable. They demonstrate the use of windows and further signal processing in order to identify different layers.

\section{REFERENCES}

Harris, F. J. (1978). On the use of windows for harmonic analysis with the discrete Fourier transforms. Proceedings of the IEEE, 66, 51-83.

Hines, M. E. and STINEHElfER, H. E. (974). Time-domain oscillographic microwave network analysis using frequency domain data. IEEE Trans. Microwave Theory Tech., 22, 276282.

Hollway, D. L. (1967). The comparison reflectometer. IEEE Trans. Microwave Theory Tech., 15, 250-259.

KING, R. J. (1978). Microwave Homodyne Systems (Peter Peregrinus Ltd., Hitchin).

MARTIN, D. J. (1980). Wave propagation in dispersive and attenuation media. A delay line simulation of plasma waves. Am. J. Phys., 48, 473-477.

Nicolson, A. M., Bennet, C. L. JR. and LAmEnSDORF, L. S. (1972). Applications of timedomain metrology to the automation of broad-band microwave measurements. IEEE Trans. Microwave Theory Tech., 20, 3-9.

Oppenheim, A. V. and Schafer, R. W. (1975). Digital Signal Processing (Prentice-Hall, Inc.).

RoBinson, L. A., WeIR, W. B. and Young, L. (1972). An RF time-domain reflectometer not in real time. IEEE Trans. Microwave Theory Tech., 20, 855-857.

SAXTON, J. A. and LANE, S. A. (1952). Electrical properties of sea water. Wireless Engineer, 29, 269-275.

SomLo, P. I. (1972). The locating reflectometer. IEEE Trans. Microwave Theory Tech., 20, 105-112.

StARK, H. and Tuteur, F. B. (1979). Modern Electrical Communications (Prentice-Hall, Inc.).

Ulriksson, B. (1981). [Report] A Reflectometer System Using Frequency Domain Data and the Fast Fourier Transform (Chalmers University of Technology).

ULRIKSSON, B. (1981). A time domain reflectometer using a semi-automatic network analyzer and the fast Fourier transform. IEEE Trans. Microwave Theory Tech., 29, 172-174.

Von Hippel, A. (1954). Dielectric Materials and Applications (The Technology Press of M.I.T. and John Wiley \& Sons, Inc.). 